\title{
Retinoic acid signaling is essential for airway smooth muscle homeostasis
}

\author{
Felicia Chen, ${ }^{1}$ Fengzhi Shao, ${ }^{1}$ Anne Hinds, ${ }^{1}$ Sean Yao, ${ }^{2}$ Sumati Ram-Mohan, ${ }^{2}$ Timothy A. Norman, ${ }^{1}$ \\ Ramaswamy Krishnan, ${ }^{2}$ and Alan Fine ${ }^{1,3}$ \\ ${ }^{1}$ The Pulmonary Center, Boston University School of Medicine, Boston, Massachusetts, USA. ${ }^{2}$ Center for Vascular Biology \\ Research, Department of Emergency Medicine, Beth Israel Deaconess Medical Center, Boston, Massachusetts, USA. \\ ${ }^{3}$ Division of Pulmonary, Critical Care, and Allergy, West Roxbury Veterans Hospital, West Roxbury, Massachusetts, USA.
}

\begin{abstract}
Airway smooth muscle (ASM) is a dynamic and complex tissue involved in regulation of bronchomotor tone, but the molecular events essential for the maintenance of ASM homeostasis are not well understood. Observational and genome-wide association studies in humans have linked airway function to the nutritional status of vitamin A and its bioactive metabolite retinoic acid (RA). Here, we provide evidence that ongoing RA signaling is critical for the regulation of adult ASM phenotype. By using dietary, pharmacologic, and genetic models in mice and humans, we show that (a) RA signaling is active in adult ASM in the normal lung, (b) RA-deficient ASM cells are hypertrophic, hypercontractile, profibrotic, but not hyperproliferative, (c) TCF- $\beta$ signaling, known to cause ASM hypertrophy and airway fibrosis in human obstructive lung diseases, is hyperactivated in RA-deficient ASM, (d) pharmacologic and genetic inhibition of the TCF- $\beta$ activity in ASM prevents the development of the aberrant phenotype induced by RA deficiency, and (e) the consequences of transient RA deficiency in ASM are long-lasting. These results indicate that RA signaling actively maintains adult ASM homeostasis, and disruption of RA signaling leads to aberrant ASM phenotypes similar to those seen in human chronic airway diseases such as asthma.
\end{abstract}

Conflict of interest: The authors have declared that no conflict of interest exists.

Submitted: February 7, 2018 Accepted: July 11, 2018 Published: August 23, 2018

Reference information: JCI Insight. 2018;3(16):e120398. https://doi.org/10.1172/jci. insight.120398.

\section{Introduction}

Airway smooth muscle (ASM), which surrounds the lumen of the bronchi, is a key regulator of bronchomotor tone. Although the precise function of mature ASM in the healthy state is not clear, ASM plays a fundamental role in the pathogenesis of chronic airway diseases such as asthma by undergoing marked phenotypic changes, including the acquisition of a hypercontractile, hyperresponsive, and profibrotic state (1). Several studies have demonstrated a strong connection between airway function and phenotypic changes in ASM. For instance, airway hyperresponsiveness (AHR), a cardinal feature of asthma defined as the exaggerated airway narrowing to constrictive stimuli, is highly associated with ASM hypercontractility (2). Likewise, persistent airflow obstruction, often observed in severe asthmatics, is closely correlated with ASM hypertrophy (3).

Homeostasis refers to any process that living tissues use to preserve stable conditions necessary for survival. Several observational studies have reported the connection between retinoic acid (RA), the biologically active metabolite of vitamin A in most tissues, to the health of the human airways. This relationship was illustrated in a large US cohort study where airway function was found to be independently and directly related to serum vitamin A level (4). A similar correlation was also established in children with respiratory tract infection-associated wheezing and adult patients with obstructive lung diseases such as asthma, chronic obstructive pulmonary disease (COPD), and cystic fibrosis (5-8). In addition, a number of genome-wide association studies (GWAS) identified a genomic locus in the RA receptor- $\beta$ ( $R A R B$ ) gene to be highly associated with airway function in healthy cohorts and patients with asthma or COPD (9-12). These observations suggest that RA signaling is involved in regulating airway function.

Vitamin A is a fat-soluble micronutrient essential for the development and maintenance of many body tissues. In target cells, vitamin A is sequentially oxidized to retinaldehyde and then irreversibly to RA, the latter reaction performed mainly by the enzymes of the aldehyde dehydrogenase family 1 , subfamily A (ALDH1A1, ALDH1A2, and ALDH1A3). The newly synthesized RA is catabolized to an inactive metabolite, transported to a nearby target cell, or moved into the nucleus, where RA binds to a heterodimer 
of transcription factors comprising a retinoic acid receptor (RARA, RARB, or RARG) and a retinoid X receptor. The ligand-receptor complex then binds to the RA response element (RARE) to activate transcription of RA target genes (13).

TGF- $\beta$ signaling has been implicated in the pathogenesis of human chronic airway diseases (14-17). It participates in airway narrowing by increasing ASM cell size and contractile protein expression, resulting in hypercontractility and hyperresponsiveness (18-23). It also mediates the expression of collagens involved in airway fibrosis through a SMAD-dependent mechanism (24). Thus, the evidence seems to suggest that TGF- $\beta$ is pathogenic in diseases characterized by ASM hypertrophy, AHR, and airway fibrosis.

Using mouse models, we have shown that prenatal RA deficiency leads to AHR in adult mice (25). The current study addresses the role of tonic RA signaling in postnatal airway health. We provide evidence that a short-term disruption of RA signaling in ASM leads to long-lasting hyperactivation of the TGF- $\beta$ pathway resulting in ASM hypertrophy, hypercontractility, AHR, and airway fibrosis. Our findings here underscore the importance of RA activity in the maintenance of structure and function of adult ASM.

\section{Results}

Disruption of $R A$ signaling in vivo for 5 days increases airway responsiveness. To investigate the effect of RA deficiency on airway function, we developed 2 approaches to block RA activity in vivo: dietary deprivation of vitamin A and pharmacologic inhibition of RA receptors. For the dietary approach, adult mice were given a vitamin A-sufficient (VAS) diet or a vitamin A-deficient (VAD) diet for 5 days $(25,26)$. For the pharmacologic approach, adult mice were fed a control (CTR) diet or a diet containing a potent pan-RA receptor inhibitor BMS493 (BMS) for 5 days $(27,28)$. To confirm downregulation of RA signaling with these 2 methods, lungs from adult mice carrying the RA activity reporter transgene RARE-lac $Z$ were collected and analyzed by real-time PCR (29). There was a significant reduction in $R A R E$-lacZ expression in the VAD and BMS (RA-deficient) lungs compared with the VAS and CTR (RA-sufficient) lungs, respectively (Figure 1A), indicating a reduction of RA activity in the lungs with either dietary (VAD) or pharmacologic (BMS) manipulation. The more pronounced RA deficiency induced by BMS in the lung is expected, since endogenous vitamin A stores in the liver and lung partially compensate for the VAD diet, while the action of BMS on RA receptors is not affected by the dietary or vitamin A stores.

Next, we used a computer-controlled piston ventilator (flexiVent, SCIREQ) to measure airway resistance in VAS, VAD, CTR, and BMS mice (30). When challenged with the bronchoconstrictor methacholine $(\mathrm{MeCh}), \mathrm{VAD}$ and BMS mice had a statistically significant increase in airway responsiveness compared with VAS and CTR mice, respectively (Figure 1, B and C). AHR was more exaggerated in the BMS lung than the VAD lung, likely reflecting a more severe functional RA deficiency in the BMS lung (Figure 1A). Airway resistance was also higher in BMS lung than the CTR lung without MeCh provocation, indicating a baseline airflow limitation in the severely RA-deficient lung (Figure $1 \mathrm{C}, 0 \mathrm{mg} / \mathrm{ml}$ of $\mathrm{MeCh} ; P<0.0001)$. These observations revealed worsening of airway function when RA signaling is disrupted in vivo for 5 days.

$R A$ deficiency results in airway remodeling characterized by increased ASM layer thickness. Airway remodeling refers to the structural changes that contribute to airflow limitation and AHR. These changes, often observed in the airways of severe asthmatics, include an increase in mucus-producing cells, extracellular matrix (ECM) deposition, and ASM mass $(31,32)$. To determine if there were structural differences between RA-sufficient and RA-deficient mouse airways, we isolated lungs from VAS, VAD, CTR, and BMS mice and performed several histologic analyses. Airway inflammation, which is a cardinal feature of asthma (33-37), was not prominent in these lungs. There were no inflammatory cells in the airways (Figure 1, D-K, H\&E data not shown). In addition, key cytokines implicated in allergic asthma such as Il4, Il5, and Ill3 were not detectable in these lungs by real-time PCR (38) (data not shown). Mucus-producing cells and mucin production were also absent in the intraparenchymal airway of these lungs (data not shown). Plastic sections revealed a thicker ASM layer in VAD and BMS airways compared with their controls (Figure 1, D-G, quantified in Supplemental Figure 1A; supplemental material available online with this article; https://doi. org/10.1172/jci.insight.120398DS1), which was due to the increase in ASM mass, as shown by the immunostaining of smooth muscle marker $\alpha$-smooth muscle actin ( $\alpha$-SMA) (Figure 1, H and I), and in airway collagen content, as demonstrated by trichrome staining (Figure 1, J and K, quantified in Supplemental Figure 1B). The expression of smooth muscle-specific genes (Acta2 and Myh11) and collagen (Colla2) was increased in RA-deficient lungs (Supplemental Figure 1C), consistent with our histologic analyses. 
A

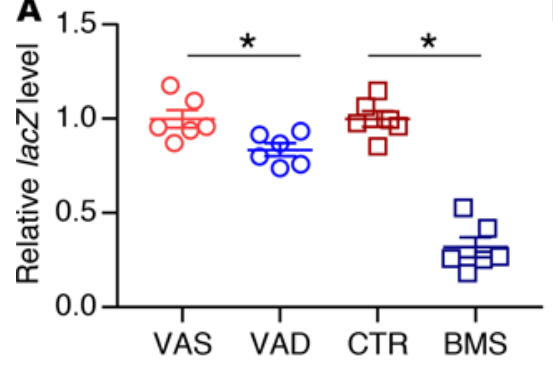

B

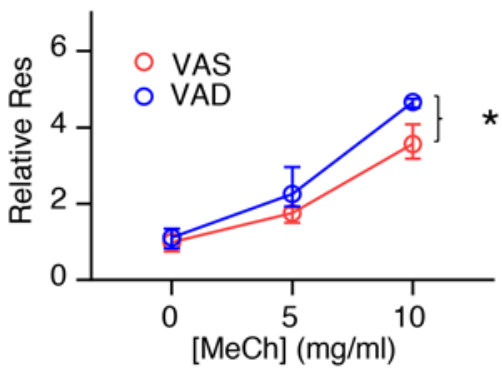

C

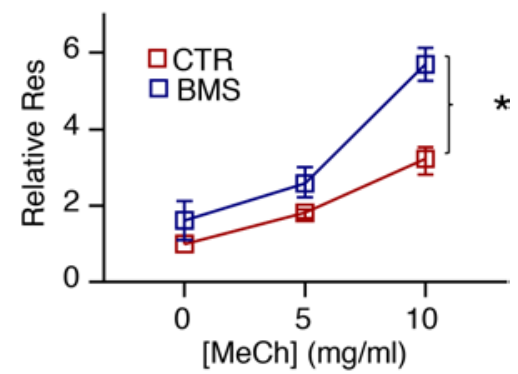

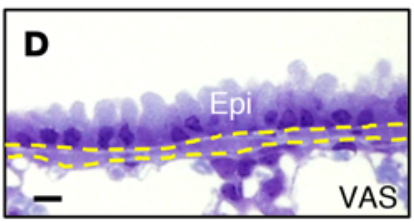
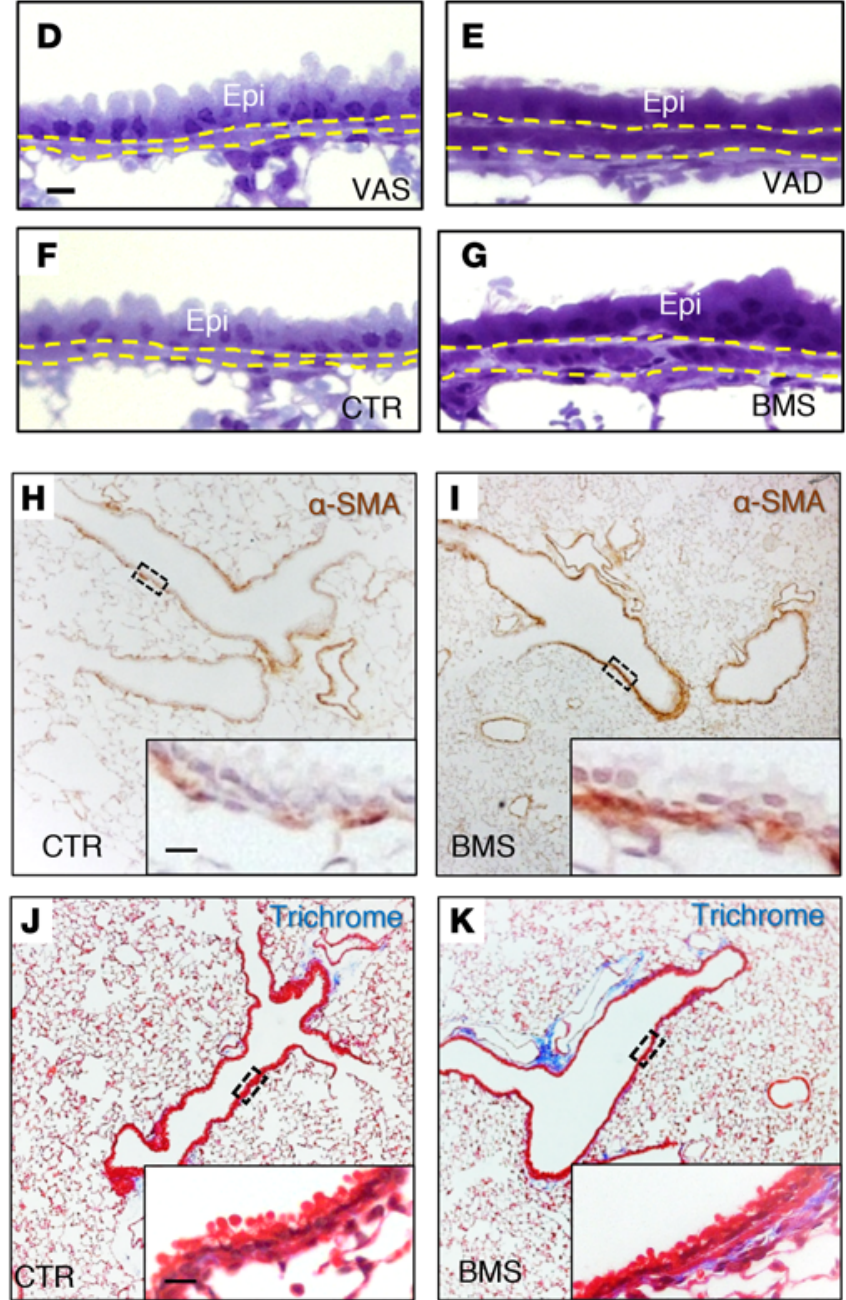
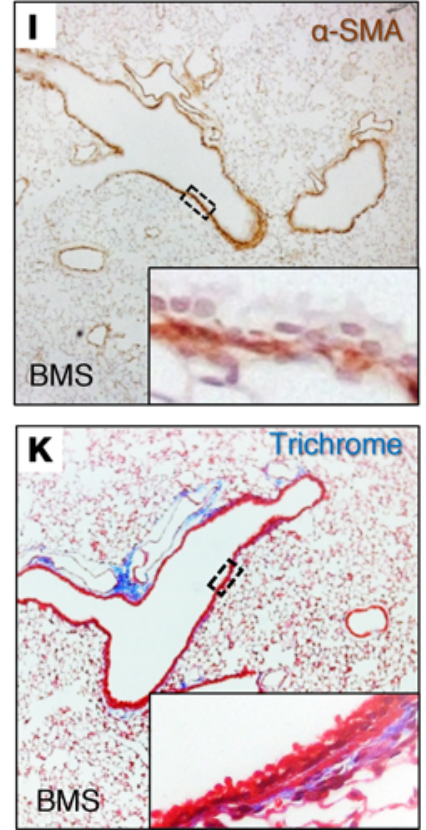

Figure 1. Disruption of RA signaling in vivo for $\mathbf{5}$ days results in airway hyperresponsiveness and airway remodeling. (A) RA activity is downregulated in RA-deficient lung as evidenced by the reduction of lacZ expression in VAD (compared with VAS) and BMS (compared with CTR) in RARE-lacZ lungs ( $n=6$ per group). (B and C) Airway resistance (Res) is higher in the VAD (B) and BMS (C) mice compared with VAS (B) and CTR (C) mice when challenged with increasing doses of the bronchoconstrictor methacholine (MeCh), indicating higher airway responsiveness in RA-deficient animals $(n=9$ per group). (D-C) Plastic sections of mouse lungs showing thicker ASM layer (delineated by yellow dotted lines) in VAD (E) and BMS (G) airways compared with VAS (D) and CTR (F) airways ( $n=3$ per group). Epi labels the airway epithelial layer. (H and I) Immunostaining of smooth muscle-specific marker $\alpha$-SMA showing an increase in ASM mass in BMS (I) airway compared with the CTR $(\mathbf{H})$ airway ( $n=3$ per group). (J and $\mathbf{K}$ ) Trichrome staining revealing increased collagen content (stained blue) in the BMS (K) airway compared to CTR (J) airway ( $n=3$ per group). Data represent the mean \pm SEM (A) or mean \pm range (B and C). Statistical significance was calculated using Student's $t$ test (A) and 2-way ANOVA (B and $\mathbf{C}) .{ }^{*} P<0.05$. Scale bars: $10 \mu \mathrm{m}(\mathbf{D}, \mathbf{H}$, and $\mathbf{J})$.

Previous reports have established a connection between protracted dietary vitamin A deficiency and airway dysfunction. The AHR in this setting was attributed to the diminished expression of cholinergic receptor, muscarinic 2 (Chrm2) and elastin (Eln) in the lung (39-42). In our models, we found no difference in the expression levels of Chrm2, cholinergic receptor, muscarinic 3 (Chrm3), or Eln between our RA-deficient and RA-sufficient lungs (Supplemental Figure 2, A-C). Perhaps the duration of RA signaling inhibition was too short to elicit these changes in expression of these genes in our models.

Altogether, our data indicate that short-term RA inhibition results in an increase in ASM mass and airway collagen deposition without bronchial inflammation or mucous hyperplasia.

$R A$ signaling is active in ASM of healthy lung. To determine whether RA signaling is active in adult ASM, we isolated a population of cells highly enriched in ASM using flow cytometry from Acta2-hrGFP;Cspg4-DsRED mouse lungs; in these lungs, ASM expressed a green fluorescent protein (hrGFP), whereas vascular smooth muscle expressed both hrGFP and a red fluorescent protein (DsRED) (Supplemental Figure 3, A and B) (43). 

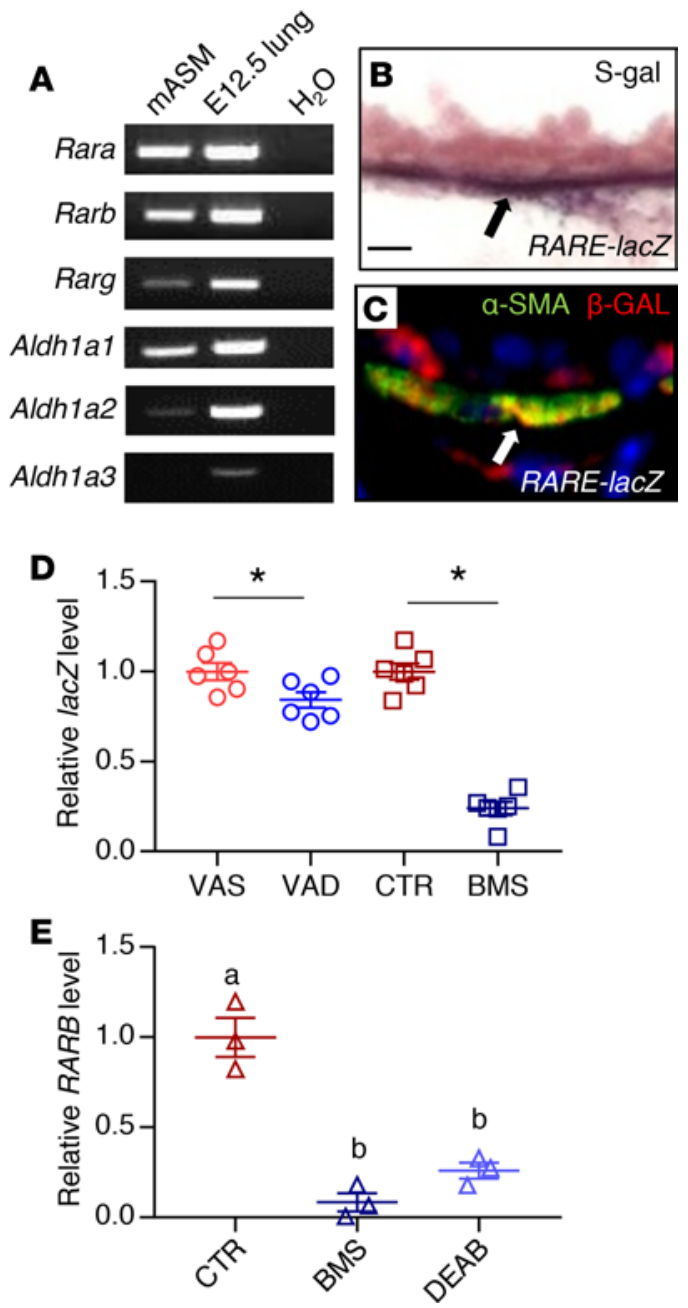

Figure 2. RA signaling is active in adult ASM. (A) PCR analysis of mASM showing the expression of RA receptors and RA-producing enzymes (Aldh1a1 and Aldh1a2). cDNA from E12.5 lung was used as a positive control $(n=3)$. (B) S-gal staining of the RA activity reporter $R A R E$-lac $Z$ lung reveals lac $Z / \beta$-gal activity in the ASM (black arrow) $(n=3)$. Scale bar: 10 $\mu \mathrm{m}$. (C) Immunostaining for lac $Z / \beta$-gal and smooth muscle-specific marker $\alpha$-SMA showing expression of lacZ/ $\beta$-gal in ASM (white arrow) ( $n=3)$. (D) lacZ expression is relatively reduced in VAD (compared with VAS) and BMS (compared with CTR) RARE-lacZ mASM, revealing downregulation of RA activity in mASM with VAD and BMS treatment ( $n=6$ per group). (E) Expression of $R A R B$, a known direct transcriptional target of RA signaling, is reduced in BMS- and DEAB-treated hASM compared with hASM cultured in CTR medium, indicating suppression of RA activity in hASM cultured in RA-deficient conditions $(n=3)$. Data represent the mean \pm SEM. Student's $t$ test was used to calculate the $P$ value in $\mathbf{D}$ and 2-way ANOVA was used for statistical analysis in $\mathbf{E}\left({ }^{*} P<0.05\right)$. Means with different letters in $\mathbf{E}$ are statistically different with Bonferronicorrected $P<0.05$.

The population of murine $\mathrm{GFP}^{+} \mathrm{DsRED}^{-}$cells (hereafter referred to as $\mathrm{mASM}$ ) expressed smooth muscle markers Acta2 and Myh11, but not the lung epithelial marker $N k x 2.1$ or the vascular smooth muscle marker Notch3 (Supplemental Figure 3C) $(44,45)$, indicating high enrichment of ASM in this population.

PCR analysis revealed the expression of RA-synthesizing enzymes (Aldh1a1 and Aldh1a2), and RA receptors (Rara, Rarb, and to a lesser extent Rarg) in mASM (Figure 2A), indicating that these cells can produce RA and turn on RA receptor-mediated signaling. Expression of RA receptor in mouse ASM was further confirmed by immunostaining for RA receptors, which revealed the expression of RARA and RARB but not RARG in the ASM (Supplemental Figure 4, A-C). S-gal staining and lacZ/ $\beta$-gal immunohistochemistry of RARE-lacZ mouse lung showed signals in the ASM cells (Figure 2, B and C) (46), supporting the idea that RA signaling is active in healthy adult ASM. Consistent with this, lacZ expression in VAD and BMS mASM isolated from triple-transgenic mouse Acta2-hrGFP;Cspg4-DsRED;RARE-lacZ was reduced compared with VAS and CTR MASM, respectively (Figure 2D), indicating that RA activity in ASM is modulated by VAD or BMS diet.

We then sought to determine whether our findings in murine ASM regarding RA signaling are paralleled in human ASM. We obtained primary, nontransformed ASM cells isolated from human donor lungs that were not suitable for transplantation (hereafter referred to as hASM cells) $(19,47)$. We chose hASM cells from 3 adult donors who had no known history of lung diseases. hASM cells are known to express RA receptors both at the mRNA and protein levels (48), which is comparable to what we had observed in mASM. When hASM cells were cultured for 24 hours in medium containing BMS or DEAB (an inhibitor of RA synthesis by blocking aldehyde dehydrogenase) (49), their expression of the RA transcriptional target $R A R B$ was lower compared with those cultured in CTR medium (Figure 2E), demonstrating that the effect of RA deficiency on ASM is direct and cell autonomous. These results confirm that RA signaling is active in both mouse and human ASM. 
Increased ASM mass seen in RA-deficient airways is due to ASM hypertrophy. An increase in ASM mass could be due to an increase in ASM cell size (hypertrophy) or number (hyperplasia). Smooth muscle hypertrophy is associated with an increase in the expression of contractile proteins and cellular contractility (50). Since the measurement of forward scatter signal (FSS) on flow cytometry allows for the discrimination of cell size (19), we compared the relative FSS of VAS, VAD, CTR, and BMS mASM. The FSS of VAD and BMS mASM were higher compared with VAS and CTR mASM (Figure 3A). The expression of $\alpha$-SMA (Acta2) and myosin heavy chain $11(M y h 11)$ was also increased in the RA-deficient mASM compared with their controls (Figure 3B). Consistent with the data from mASM, the BMS- and DEAB-cultured hASM cells were larger (Figure $3 C$ ) and expressed higher levels of the smooth muscle contractile proteins $\alpha$-SMA (protein product of $A C T A 2)$ and transgelin (SM-22 $\alpha$, another smooth muscle marker) than those cultured in control medium (51) (Figure 3, D and E).

To assess the mechanical consequences of ASM hypertrophy due to RA deficiency, we measured the intrinsic contractility of hASM cells from the 3 adult donors cultured for 24 hours in CTR, BMS, and DEAB media (52). The contractile force generated by hASM cells cultured in RA-deficient conditions (BMS and DEAB) was higher than CTR hASM (Figure 3F). Overall, these findings show that inhibition of RA signaling in ASM leads to ASM hypertrophy and hypercontractility.

To determine if there were more abundant ASM cells in RA-deficient airways, we first performed immunostaining for Ki-67, a cellular marker of proliferation (53), and found no expression in VAS, VAD, CTR, and BMS ASM layers (Figure 3, G and H), indicating the absence of proliferation in RA-sufficient or -deficient ASM. Next, we used a double-transgenic mouse (Myh11-creERT2;mT/mG), which allows for prelabeling of smooth muscle lineage with a membrane-bound GFP before CTR or BMS diet, to assess the potential of non-ASM populations to differentiate into ASM in RA-deficient conditions (54-57). The high efficiency and smooth-muscle specificity of the recombination events were evaluated by immunostaining for both GFP and $\alpha$-SMA after tamoxifen injection in VAS and CTR mice. If non-smooth muscle cells contributed to the ASM population, such cells would be lineage negative (GFP negative), but $\alpha$-SMA positive. In both CTR and BMS Myh11-creERT2;mT/mG lungs, we found that all $\alpha$-SMA-positive ASM cells were also GFP positive (Figure 3, I and J), suggesting no contribution from non-ASM lineages to ASM population regardless of RA status. Therefore, we conclude that the increase in ASM mass observed in RA deficiency is not due to increase in ASM cell number but is due to increase in ASM cell size.

TGF- $\beta$ signaling is hyperactivated in RA-deficient ASM. We then applied gene expression microarray analysis to determine the molecular targets of RA in ASM. Transcriptional profiles of mASM (from VAS, VAD, CTR, and BMS mice) and hASM cells from 3 adult donors (cultured in CTR or BMS media) were generated using Affymetrix array chips. To identify direct RA targets with high specificity, we included only genes whose expression was induced by RA deficiency in both mASM and hASM cells in the final list of RA targets. This list was then input into Expression Analysis Systematic Explorer (EASE) software to determine enriched gene sets $(58,59)$. Of the gene sets enriched by RA deficiency, collagens, ECM constituents, and TGF- $\beta$ ligands ("TGF- $\beta$ receptor binding") were most highly represented (Table 1 ), raising the possibility that RA deficiency induces excessive activation of TGF- $\beta$ signaling in ASM.

We next performed immunostaining for phospho-SMAD2 (p-SMAD2), an intracellular marker of TGF- $\beta$ receptor activation, in VAS, VAD, CTR, and BMS lungs (60). There was little to no signal in the ASM layer of VAS and CTR lungs (Figure 4A; VAS data not shown), but signals were present in ASM of VAD and BMS lungs (Figure 4B; VAD data not shown). The ratio of p-SMAD2 signal to total SMAD2 (tSMAD2) signal was significantly upregulated in BMS-treated and DEAB-treated hASM compared with CTR hASM cells (Figure $4 C)$, supporting hyperactivation of the TGF- $\beta$ pathway in RA-deficient ASM. We also found a significant increase in the expression of the TGF- $\beta$ target Colla2 mRNA in VAD and BMS mASM (Figure 4D) and BMS- and DEAB-treated hASM (Figure 4E) compared with their controls. TGF- $\beta 1$ treatment recapitulated the phenotypes observed in RA-deficient hASM including the induction of p-SMAD2, COL1A2, and ACTA2, and the increase in contractile force generation relative to untreated CTR cells (Figure 5, A-D). Interestingly, both Tgfb1/ TGFB1 and Tgfb3/ TGFB3 ligands were upregulated in RA-deficient ASM in our microarray analysis (Table 1, "TGF- $\beta$ receptor binding") and real-time PCR (Supplemental Figure 5, A and B). TGF- $\beta 1$ treatment also appeared to suppress $R A R B$ (Supplemental Figure $5 \mathrm{C}$ ), suggesting further suppression of RA signaling when the TGF- $\beta$ pathway is activated. These data point to the induction of TGF- $\beta$ ligands as a potential cause of TGF- $\beta$ hyperactivity in RA-deficient ASM. Overall, our observations here strongly favor TGF- $\beta$ as a mediator of the aberrant phenotype seen in RA-deficient ASM. 
A

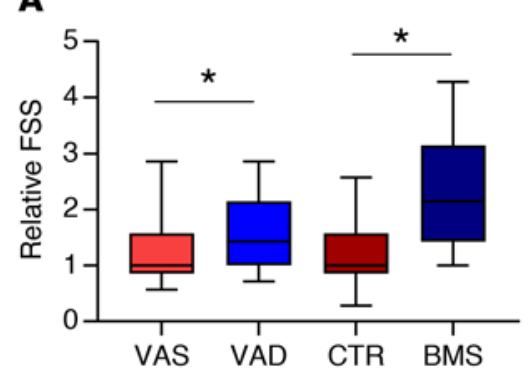

C

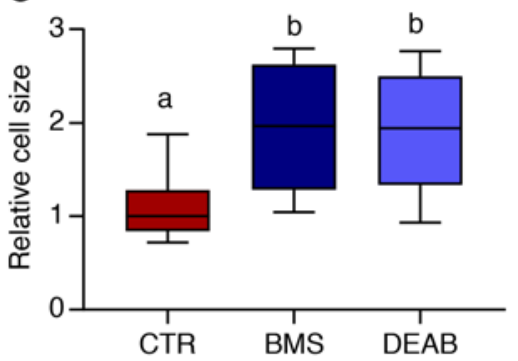

$\mathbf{E}$

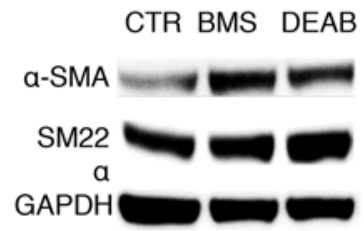

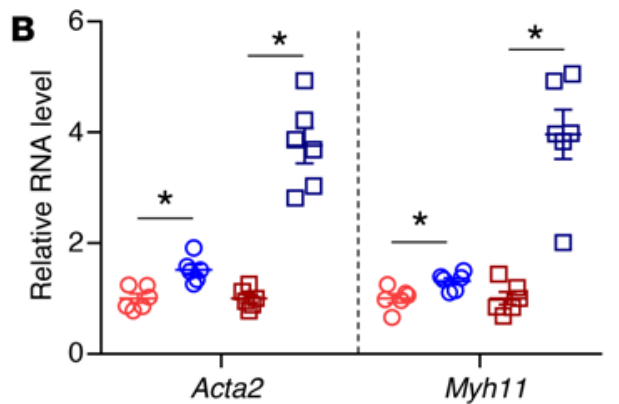

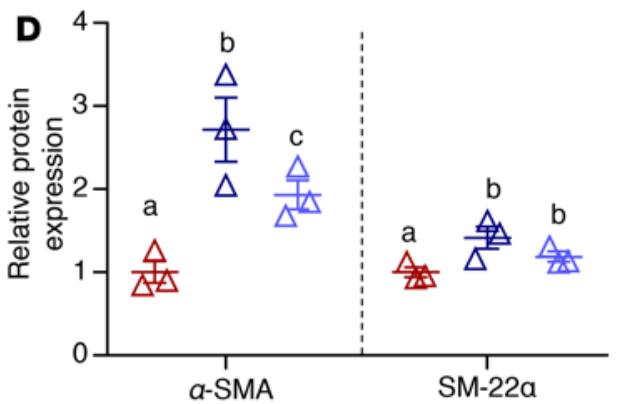

$\mathbf{F}$

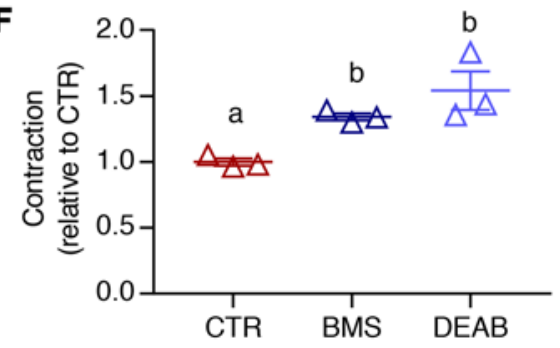

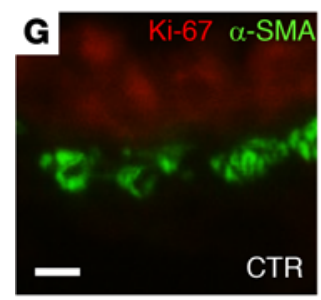
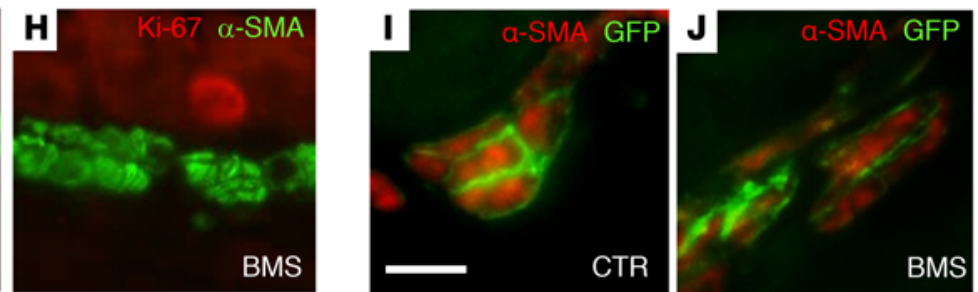

Figure 3. RA deficiency in mice results in ASM hypertrophy. (A) Forward scatter signals (FSS) of mASM showing higher FSS in RA-deficient mASM (VAD and BMS) compared with RA-sufficient mASM (VAS and CTR, respectively), suggesting larger mASM cell size in RA-deficient lungs ( $n=3$ ). (B) Expression of smooth muscle markers Acta2 and Myh11 is increased in VAD and BMS mASM cells compared with their controls (VAS and CTR, respectively), further supporting ASM hypertrophy in RA-deficient mASM ( $n=6$ per group). (C) Size of hASM cells is increased when cultured in RA-deficient conditions (BMS and DEAB) compared with those cultured in RA-sufficient conditions (CTR) ( $n=3$ ). (D) Expression of smooth muscle $\alpha$-SMA and SM-22 $\alpha$ is significantly higher in BMS- and DEAB-treated hASM compared with CTR-treated hASM, indicating hASM hypertrophy when RA signaling is inhibited ( $n=3$ ). (E) Representative Western blot of data displayed in $\mathbf{D}(n=3$; blots shown are from donor 1$)$. ( $F)$ Intrinsic cellular contraction of RA-deficient hASM (BMS, DEAB) is higher compared with RA-sufficient hASM (CTR), indicating greater hASM contractility when cultured in RA-deficient conditions ( $n=3)$. ( $\mathbf{C}$ and H) Immunostaining of Ki-67, a marker of cell proliferation, and $\alpha$-SMA showing no Ki-67 signal in CTR ASM (C) or BMS ASM (H) in mouse ( $n=3)$. (I and J) Immunostaining of $\alpha$-SMA and smooth muscle lineage (GFP) in tamoxifen-injected Myh11-CreERT2;mT/mG mice showing membranous GFP signal in all $\alpha$-SMA+ cells in both CTR and BMS airways, arguing against non-ASM contribution $(n=3)$. Data in $\mathbf{A}$ and $\mathbf{C}$ are presented as box-and-whisker plot (see Statistics in Methods section for description). Data in $\mathbf{B}, \mathbf{D}$, and $\mathbf{F}$ represent the mean \pm SEM. Student's $t$ test was used to calculate $P$ values in $\mathbf{A}$ and $\mathbf{B}$ ( ${ }^{*} P$ $<0.05)$. Two-way ANOVA was used for statistical analysis in $\mathbf{C}, \mathbf{D}$, and $\mathbf{F}$, where Bonferroni's correction was applied to adjust $P$ values for multiple comparisons (means with different letters are significantly different, $P<0.05$ ). Scale bars: $10 \mu \mathrm{m}$ ( $\mathbf{D}$ and $\mathbf{F})$.

Inhibition of TGF- $\beta$ signaling in ASM prevents the phenotypic changes induced by RA deficiency. If a role of endogenous RA is to maintain a normal ASM phenotype by preventing excessive activation of TGF- $\beta$ signaling, then inhibition of TGF- $\beta$ in ASM should ameliorate the abnormal phenotype induced by RA deficiency. To test this in hASM, we used a selective TGF- $\beta$ receptor 1 kinase inhibitor (SB431542; hereafter referred to as SB) to block TGF- $\beta$ signaling in hASM (61). Adding SB decreased the p-SMAD2/tSMAD2 
Table 1. Top gene categories enriched by RA deficiency in both human and mouse ASM

\begin{tabular}{|c|c|c|}
\hline Gene Category & EASE score & Human Gene Symbol \\
\hline collagen & $2.09 \times 10^{-8}$ & COL10A1, COL15A1, COL3A1, COL4A1, COL4A2, COL5A1, COL5A2, COL6A3, COL7A1 \\
\hline ECM structural constituent & $3.92 \times 10^{-7}$ & COL10A1, COL15A1, COL3A1, COL4A1, COL4A2, COL5A1, COL5A2, COL6A3, COL7A1, FBN1, WNT7B \\
\hline extracellular & $1.19 \times 10^{-5}$ & $\begin{array}{c}\text { ADAM15, CILP, CLU, COL10A1, COL15A1, COL3A1, COL4A1, COL4A2, COL5A1, COL5A2, COL6A3, COL7A1, } \\
\text { CRLF1, FBN1, FGF13, CSN, IL16, INHBA, INHBE, ITGB3, LIF, LOXL1, MFAP4, MMP16, NUCB2, SERPINE2, } \\
\text { TCFB1, TGFB3, TCFBI, TNC, TUFT1, WNT5A, WNT7B, ZG16 }\end{array}$ \\
\hline TGF- $\beta$ receptor binding & $3.00 \times 10^{-3}$ & INHBA, TGFB1, TCFB3 \\
\hline integral to membrane & $7.60 \times 10^{-3}$ & $\begin{array}{l}\text { ACE, ADAM12, ADAM15, AOC3, AOP1, ATP10A, BET1, BNIP3, C18ORF1, CAV2, CD81, CDIPT, CELSR1, DCT, } \\
\text { FZD8, GRIA3, HAS2, HS3ST3A1, IL17B, ITCB3, KCNH1, KDELR3, LRRC15, MGAT5, MMP16, MRC2, PCDHA9, } \\
\text { PGRMC2, PICM, POMT2, PTK7, PTPRN, RABAC1, RNF121, SC5DL, SCN7A, SCN9A, SEL1L, SIRPB1, } \\
\text { SLC19A2, SLC1A4, SLC25A20, SLC3A2, SLC4A2, SLC7A11, SOLE, TAS1R3, TXNIP, UCP2, ZMPSTE24 }\end{array}$ \\
\hline
\end{tabular}

ECM, extracellular matrix; EASE, Expression Analysis Systematic Explorer.

ratio, the levels of collagen (COL1A2) and smooth muscle markers (ACTA2 and MYH11), and contractility in RA-deficient and TGF- $\beta 1$-treated hASM (Figure 5, A-D).

To examine this further in vivo, we crossed mice homozygous for TGF- $\beta$ receptor 2 (Tgfbr2) in which exon 4 was flanked by $\operatorname{LoxP}$ sites (Tgfbr $\left.2^{f / f}\right)$ with $M y h 11$-creERT2 mice, allowing for selective inhibition of TGF- $\beta$ receptor-mediated signaling in smooth muscle after tamoxifen injection $(62,63)$. After tamoxifen administration, we placed these mice on a CTR or a BMS diet for 5 days (TAM-CTR and TAM-BMS, respectively). Vehicle-injected (corn oil) Myh11-creERT2; Tgfbr $2^{f / f l}$ littermates fed with a CTR or a BMS diet for 5 days were used as additional controls (OIL-CTR and OIL-BMS, respectively). As expected, p-SMAD2 labeling was present in nuclei of OIL-BMS ASM (Figure 6B), but not in the OIL-CTR or the TAM-CTR ASM (Figure 6, A and C). However, there was no p-SMAD signal in TAM-BMS ASM, despite 5 days of BMS treatment (Figure 6D). ASM layer thickness was notably increased in the OIL-BMS airways but not in the TAM-BMS airways (Figure 6E). Remarkably, the higher airway resistance and airway hyperresponsiveness induced by BMS was prevented in TAM-BMS mice (Figure 6F). These data demonstrate that inhibition of TGF- $\beta$ signaling in ASM blocks the aberrant phenotypic changes associated with RA deficiency, further confirming TGF- $\beta$ as a downstream target of RA deficiency in ASM.

Short-term $R A$ deficiency induces long-lasting changes in ASM phenotype. Finally, we determined whether the changes in RA-deficient ASM were reversible by restoring RA signaling. To examine this, we gave adult WT or RARE-lacZ mice 5 days of CTR or BMS diet followed by 28 days of a VAS diet (washout) (Figure 7A). These mice were designated CTR-28R and BMS-28R, respectively. lacZ expression levels in RARE-laZ CTR-28R and BMS-28R lungs were similar, indicating restoration of RA activity after 28 days of VAS diet in the BMS-28R lung (Figure 7B). Myh11 and Colla2 expression remained elevated in the BMS-28R lung, but not to the extent seen immediately following 5 days of BMS diet (Figure 7B, compared with Supplemental Figure 1C). Similarly, BMS-28R mice continued to show AHR with MeCh challenge, though they did not have the increased baseline airway resistance seen in BMS mice (Figure 7C, compare right with left panel). Immunostaining of p-Smad2 revealed persistent signals in the BMS-28R ASM (Figure 7, D and E). While the impact appears to wane over time, these data show that the RA-deficient phenotype persists in ASM weeks after the restoration of RA activity.

\section{Discussion}

Vitamin A deficiency is one of the most common nutritional deficiencies in the world (64). It is often recognized as a problem in the under-developed countries, but it is also present in the resource-rich regions. People at risk of vitamin A deficiency include those with malnutrition, obesity, chronic illness, alcoholism, and malabsorption syndromes (e.g., cystic fibrosis, celiac disease, inflammatory bowel disease) (65-67). Numerous cross-sectional studies have linked the nutritional status of vitamin A to airway function. Importantly, even a small decrease in serum vitamin A level is associated with worse airway function (4). The strong correlation 

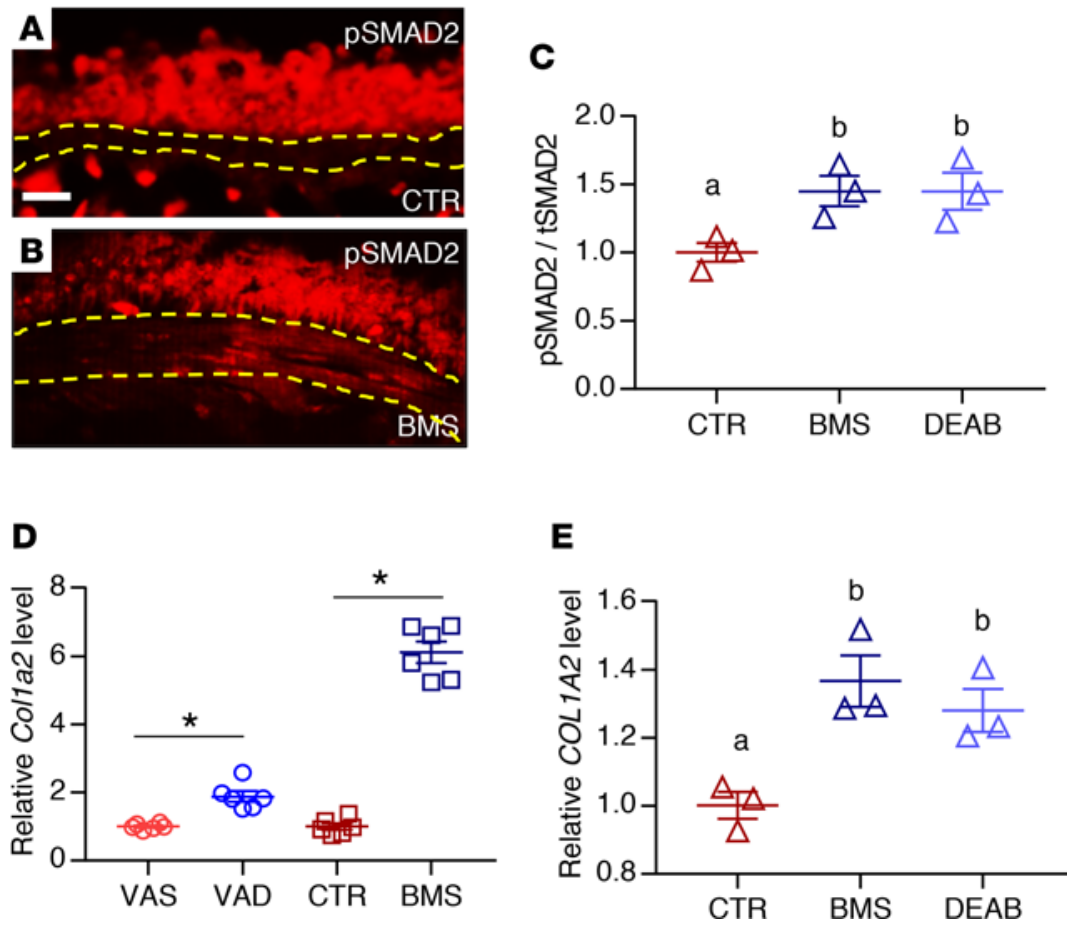

Figure 4. TGF- $\boldsymbol{\beta}$ is activated in RA-deficient ASM. (A and $\mathbf{B}$ ) Immunostaining of phospho-SMAD2 (p-SMAD2) showing signals within the ASM layer of BMS (B) but not CTR (A) airway, indicating activation of TCF- $\beta$ signaling in RAdeficient mouse ASM ( $n=3$ per group). Scale bar: $20 \mu \mathrm{m}$. (C) p-SMAD2 to total SMAD2 (tSMAD2) ratio is increased in BMS- and DEAB-treated hASM compared with hASM cultured in CTR medium, indicating higher level of TCF- $\beta$ activity in the RA-deficient hASM $(n=3)$. (D) Expression of COL1A2, a transcriptional target of TCF- $\beta$ signaling, is increased in VAD mASM (compared with VAS MASM) and BMS MASM (compared with CTR MASM) ( $n=3$ per group). (E) COL1A2 production is increased with BMS- and DEAB-treated hASM compared with CTR hASM $(n=3)$. Data represent the mean \pm SEM. Student's $t$ test was used to calculate $P$ values in $\mathbf{D}\left({ }^{*} P<0.05\right)$. Two-way ANOVA was used for statistical analysis in $\mathbf{C}$ and $\mathbf{E}$ where $P$ values were adjusted by Bonferroni's correction (means with different letters are significantly different with $P<0.05$ ).

between airway function and a variant allele of the RA receptor- $\beta(R A R B)$ gene revealed by GWAS studies raises the possibility that alteration in RA receptor activity, independent of vitamin A availability, could affect airway health (9-12). Here, we show that the maintenance of normal ASM phenotype is an active process and that ongoing RA receptor-mediated signaling is critical for proper ASM structure and function.

A link between vitamin A deficiency and AHR had been reported in rats exposed to VAD diet for a prolonged period. The AHR was attributed to the downregulation of cholinergic receptor, muscarinic 2 (Chrm2) and decreased elastance in the airspaces, but no structural alteration in the airway was reported (39-42). Our methodology is different because we limited our window of vitamin A/RA deficiency to a brief period to detect the early effect of RA deficiency (5 days in live mice, 24 hours in hASM cultures). We found no difference in airspace morphology, elastance, or expression levels of Chrm2 or elastin (ELN) mRNA between RA-sufficient and -deficient lungs. The discrepancy is likely due to the length of vitamin A/RA deficiency. Other studies have reported alteration in immune responses and AHR to pharmacologic doses of RA in allergen-sensitized mice with conflicting results $(68,69)$. However, comparing our observations to these previous reports is difficult since we did not induce allergic inflammation in our models and we were evaluating the effect of RA deficiency, not RA excess, on the airway structure and function.

TGF- $\beta$ signaling has been implicated in the pathogenesis of several airway diseases involving bronchoconstriction and airway remodeling. Interestingly, the ASM phenotype induced by TGF- $\beta$ activation is very similar to that caused by RA deficiency. TGF- $\beta$ promotes the differentiation of ASM cells into a more contractile phenotype (70), induces AHR in hASM in culture (23), and mediates the expression of profibrotic genes such as collagens (24). We find that TGF- $\beta$ effector and targets are upregulated in our RA-deficient models, and blockade of TGF- $\beta$ activity in ASM averts the aberrant changes induced by RA deficiency. These findings strongly implicate TGF- $\beta$ hyperactivation as the mediator of RA deficiency in ASM. 
A

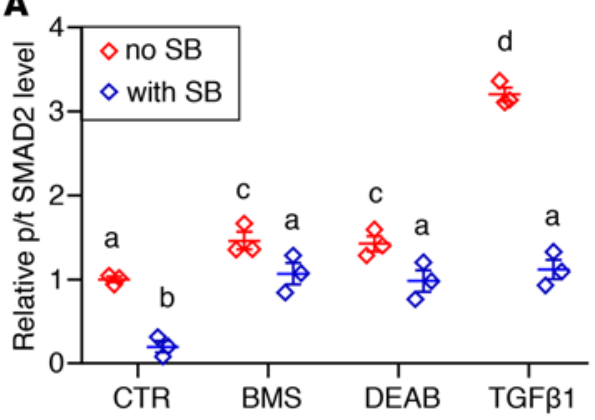

C

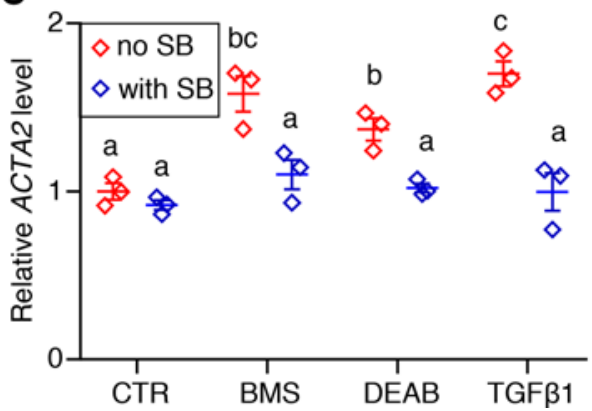

B

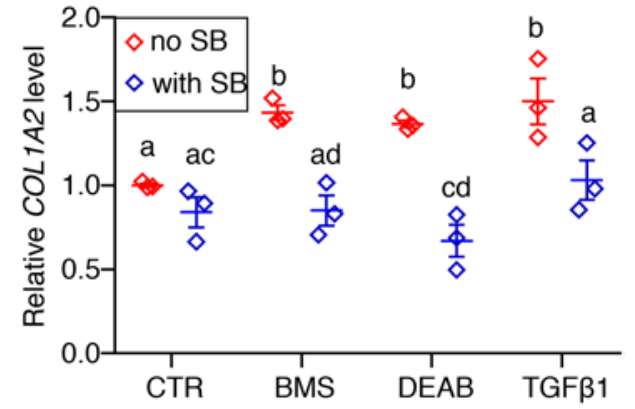

D

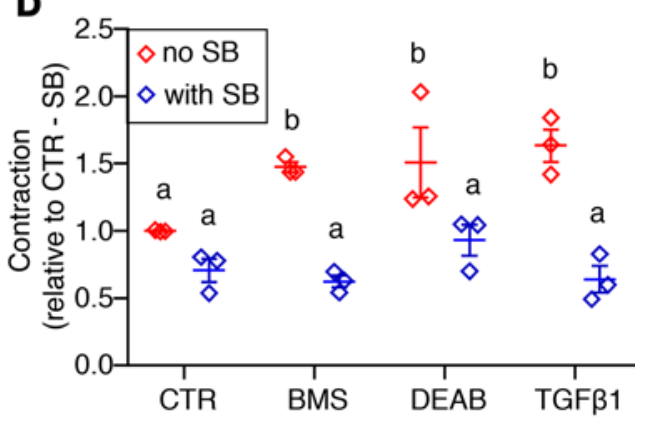

Figure 5. Inhibition of TGF- $\beta$ receptor-mediated signaling prevents phenotypic changes caused by RA deficiency in cultured human ASM. hASM cells from 3 donors were cultured with control medium (CTR), pan-RA receptor antagonist (BMS), RA synthesis inhibitor (DEAB), and TGF- $\beta 1$ with or without the TCF- $\beta$ type I receptor kinase inhibitor SB. (A) Phospho-SMAD2 ( $p$-SMAD2) to total SMAD2 (tSMAD2) ratio in BMS- and DEAB-treated hASM is reduced when hASM is also treated with SB, suggesting a decrease in TCF- $\beta$ activity in RA-deficient hASM with TCF- $\beta$ receptor blockade ( $n=$ 3). (B and C) Expression of TCF- $\beta$ target COL1A2 (B) and smooth muscle-specific marker ACTA2 (C) in BMS- and DEABtreated hASM is lowered by adding SB to the culture medium, showing that RA-deficiency-induced changes in hASM are ameliorated with inhibition of TGF- $\beta$ signaling $(n=3)$. (D) Hypercontractility resulting from BMS and DEAB treatment is diminished by cotreatment with SB $(n=3)$. hASM cells cultured with TCF- $\beta 1$ with or without SB are included as additional controls and for assessing the efficacy of SB in downregulating TCF- $\beta$ signaling. All measured parameters are graphed relative to non-SB-treated CTR hASM (CTR/-SB). Data represent the mean \pm SEM. Two-way ANOVA was used for statistical analysis. Bonferroni's correction for multiple comparisons was applied to adjust $P$ values. Means with different letters are significantly different with $P<0.05$.

Though TGF- $\beta$-related genes are well represented in our transcriptional profiling experiments, the gene most induced by RA deficiency in mASM, IL-17B (Il17b), is not known to be associated to TGF- $\beta$. Il17b is increased 14-fold in BMS over CTR mASM and 3-fold in VAD over VAS mASM. Unlike its better-known family member IL-17A $(71,72)$, the function of IL-17B is less unclear. One report showed that IL-17B is protective against mucosal inflammation in a murine allergen-induced asthma model (73). However, since inflammation is not a prominent feature in our models, it is difficult to apply the finding of the study to our results.

We were surprised to discover that the changes in ASM induced by transient RA deficiency were sustained for over 4 weeks, despite the restoration of normal RA activity. Two processes may have contributed to this finding. First, the TGF- $\beta$ ligands 1 and 3 are autoinduced in ASM by the activation of the TGF- $\beta$ pathway, a phenomenon that has been reported in both normal and transformed cell lines $(74,75)$. Second, TGF- $\beta$ signaling, once activated, appears to downregulate RA signaling, as suggested by the downregulation of the RA receptor $R A R B$ in TGF- $\beta 1$-treated hASM. The combination of these processes favors the persistent activation of the TGF- $\beta$ pathway, which may explain the long-lasting effect of short-term RA deficiency.

In summary, disruption of RA signaling in ASM leads to increased TGF- $\beta$ ligand production followed by hyperactivation of the TGF- $\beta$ pathway. This results in phenotypic changes in ASM characterized by hypertrophy, hypercontractility, and increased collagen production and deposition, ultimately leading to airway remodeling, hyperresponsiveness, and airflow obstruction. Autoinduction of TGF- $\beta$ ligands and suppression of RA signaling by TGF- $\beta$ activation likely perpetuate the pathologic process (Figure 8). Overall, our findings demonstrate that RA signaling maintains ASM homeostasis by preventing excessive activation of the TGF- $\beta$ pathway. We conclude that tonic RA signaling is critical for postnatal airway health. 

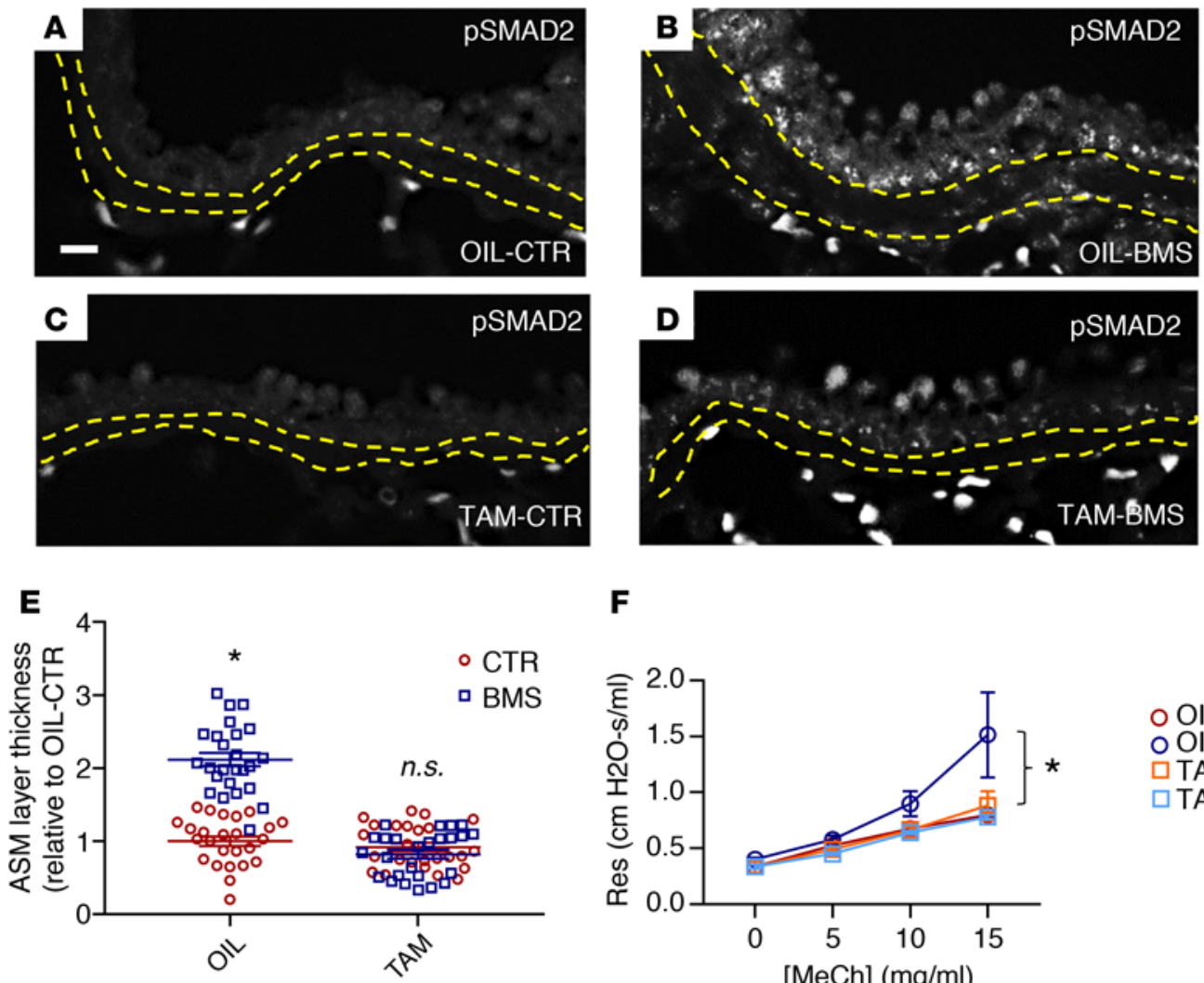

$\mathbf{F}$
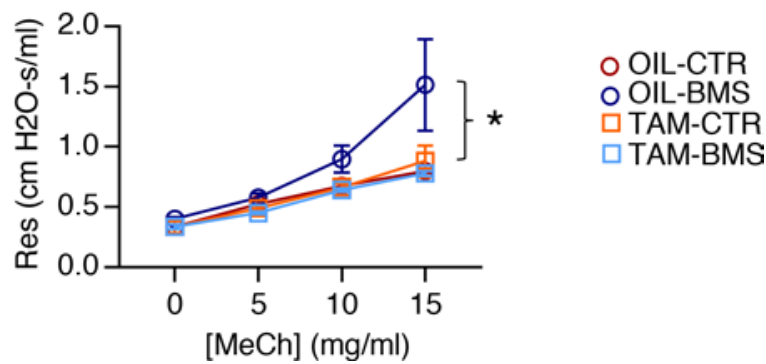

Figure 6. Deletion of TGF- $\beta$ receptor 2 (Tgfbr2) in mouse smooth muscle in vivo prevents the increase in Col1a2 expression, ASM layer thickness, and airway responsiveness induced by BMS. Myh11-CreERT2; Tgfbr $2^{f / f l}$ transgenic mice were injected with tamoxifen to delete TCF- $\beta$ receptor 2 (Tgfbr2) in the smooth muscle and then given 5 days of CTR or BMS diet (TAM-CTR, TAM-BMS). Corn oil-injected littermates with the same genotype with 5 days of CTR and BMS were used as control (OIL-CTR, OIL-BMS). (A-D) Immunostaining of phospho-SMAD2 (p-SMAD2) showing signals within the ASM layer of OIL-BMS (B) but not in OIL-CTR (A) airways, similar to what is observed in CTR and BMS airways in WT mice. Scale bar: $20 \mu \mathrm{m}$. Deletion of Tgfbr2 in smooth muscle eliminates p-SMAD2 signals in TAM-CTR (C) and TAM-BMS (D) airways ( $n=3$ per group). Yellow lines delineate the margin of the ASM layer. (E) Morphometric analysis showing a thicker ASM layer in OIL-BMS airways compared with OIL-CTR airways. There was no significant difference in the thickness of the ASM layer among OIL-CTR, TAM-CTR, and TAM-BMS airways ( $n=3$ mice per group; 8-9 airways per mouse). (F) Airway resistance (Res) is higher in the OIL-BMS group at baseline and with methacholine (MeCh) challenge compared with OIL-CTR, TAM-CTR, and TAM-BMS. There is no significant difference in airway responsiveness between OIL-CTR, TAM-CTR, and TAM-BMS mice ( $n=6$ per group). Data represent the mean \pm SEM (E) or mean \pm range $(\mathbf{F})$. Two-way ANOVA was used for statistical analysis. Bonferroni's correction for multiple comparisons was applied to adjust $P$ values. ${ }^{*} P<0.05$, n.s., $P \geq 0.05$.

\section{Methods}

RARE-lacZ reporter, Acta2-hrGFP reporter, Cspg4-DsRED reporter, $m T / m G$ reporter, Myh11-CreERT2 transgenic, and $T g f b r 2^{f l f l}$ mice. These mice were characterized previously and identified by PCR genotyping. The transgenic RARE-lacZ mouse expresses the $\beta$-galactosidase (lacZ) gene under the control of RARE. RA signaling is indicated by $\beta$-galactosidase activity (29). These mice were purchased from the Jackson Laboratory (stock 008477, CD1 background). Acta2-hrGFP transgenic mice carry a transgene of green fluorescent protein (hrGFP) under the control of the Acta2 promoter/enhancer. They were generated in Alan Fine's lab (mixed CD1/C57BL background) (43). Cspg4-DsRED transgenic mice express a red fluorescent protein (DsRED) under the control of the mouse Cspg4 promoter/enhancer (76). These were obtained from the Jackson Laboratory (stock 008241, mixed FVB/C57BL background). Myh11. CreERT2 mice were purchased from the Jackson Laboratory (stock 019079). These carry a transgene containing the Cre recombinase and estrogen receptor fusion gene driven by the promoter/enhancer of Myh11. The transgene was reportedly inserted into the $\mathrm{Y}$ chromosome $(55,56)$ and is kept in a mixed FVB/C57B background. $m T / m G$ mice (Jackson Laboratory, stock 007576, C57BL background) contain a cell membrane-targeted, 2-color fluorescent Cre-reporter in the ROSA locus. These mice express TdTomato (mT) fluorescent protein in non-Cre recombinase-expressing cells and GFP (mG) fluorescent protein in Cre-expressing cells (57). Tgfbr $2^{f / f l}$ mice (Jackson Laboratory, stock 012603, C57BL background) possess loxP sites flanking exon 4 of the Tgfbr2 gene (62). All experiments were performed 
A

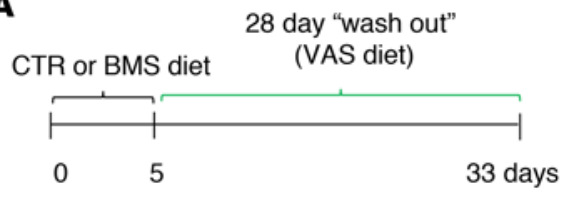

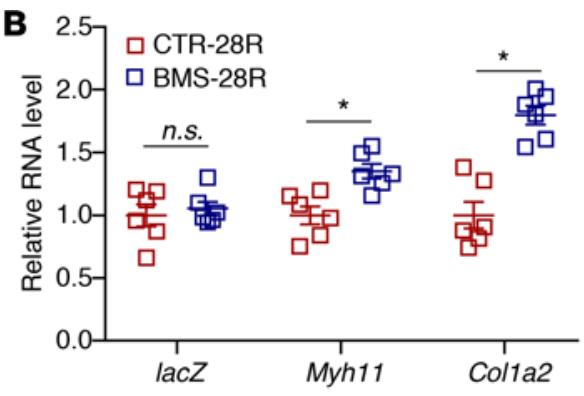
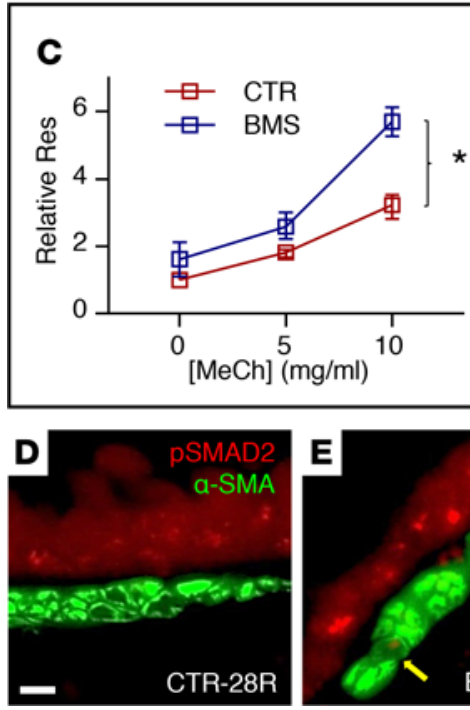

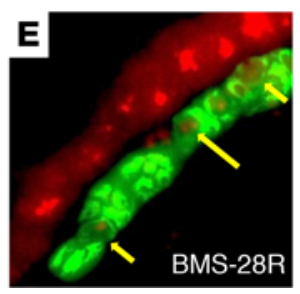

Figure 7. Consequences of RA deficiency in ASM are long-lasting despite restoration of RA activity. (A) Diagram of the experimental design. Adult mice were given 5 days of CTR and BMS diet followed by 28 days of vitamin A-sufficient diet (CTR-28R and BMS-28R, respectively). (B) mASM sorted from Acta2-hrGFP;Cspg4-DsRED;RARE-lacZ lungs shows a persistent increase in Myh11 and Col1a2 expression in BMS-28R mASM compared with CTR-28R, though RA activity, as demonstrated by the level of RARE-lacZ (lacZ) expression, is not different between CTR-28R and BMS-28R lungs ( $n$ = 6 per group). (C) Airway responsiveness in BMS-28R mice (right panel) is better compared with BMS mice (left panel), but airway resistance (Res) remains significantly higher with methacholine (MeCh) challenge compared with that of the CTR-28R mice, indicating residual AHR despite restoration of RA activity ( $n=9$ per group). Scale bar: $10 \mu \mathrm{m}$. (D and E) Immunostaining of p-SMAD2 and $\alpha$-SMA showing persistent p-SMAD2 signals in BMS-28R ASM despite 28-day washout period (yellow arrows) ( $n=3$ per group). Data represent the mean \pm SEM (B) or mean \pm range (C). Student's $t$ test was used to calculate $P$ values in B. Two-way ANOVA was used to compare the curves in $\mathbf{C}$. ${ }^{*} P<0.05$, n.s., $P \geq 0.05$.

in mice between 8 and 12 weeks of age. In experiments using Myh11-CreERT2 mice, only male mice were used because the transgene was inserted into the $\mathrm{Y}$ chromosome. In other experiments, both male and female mice were used. No gender-specific differences were noted other than the baseline airway resistance measurement on flexiVent (data not shown). Comparisons were made among age- and gender-matched animals only. When possible, only littermates were used for comparative experiments.

Dietary and pharmacologic manipulation of $R A$ activity in vivo and in vitro. Adult male mice were given 5 days of VAS diet (20 IU of vitamin A per gram of chow; Envigo, TD.91280), VAD diet (Envigo, TD.86143) $(25,26)$, powdered chow containing $5 \mu \mathrm{g} / \mathrm{g}$ of BMS (BMS493, a pan-RA receptor inhibitor; Sigma-Aldrich, B6688) $(27,28)$, or control powder chow mixed with vehicle (CTR). For the rescue experiments, 5 days of CTR or BMS diet was followed by 28 days of standard VAS food (CTR-28R, BMS-28R). All mice were given standard VAS diet before initiating dietary or pharmacologic manipulation of RA activity in vivo.

Flow cytometry of live murine ASM cells. Isolation of ASM cells by flow cytometry was described previously (43). Cell suspensions were obtained by digesting Acta2-hrGFP;Cspg4-DsRED mouse lungs with a Lung Dissociation Kit (Miltenyi Biotec, 130-095-927). mASM cells (hrGFP+DsRED ${ }^{-}$) were collected using a Mo-Flo cell sorter (Beckman Coulter). FlowJo software (Tree Star) was used to analyze the data from flow cytometry. 
Homeostsis

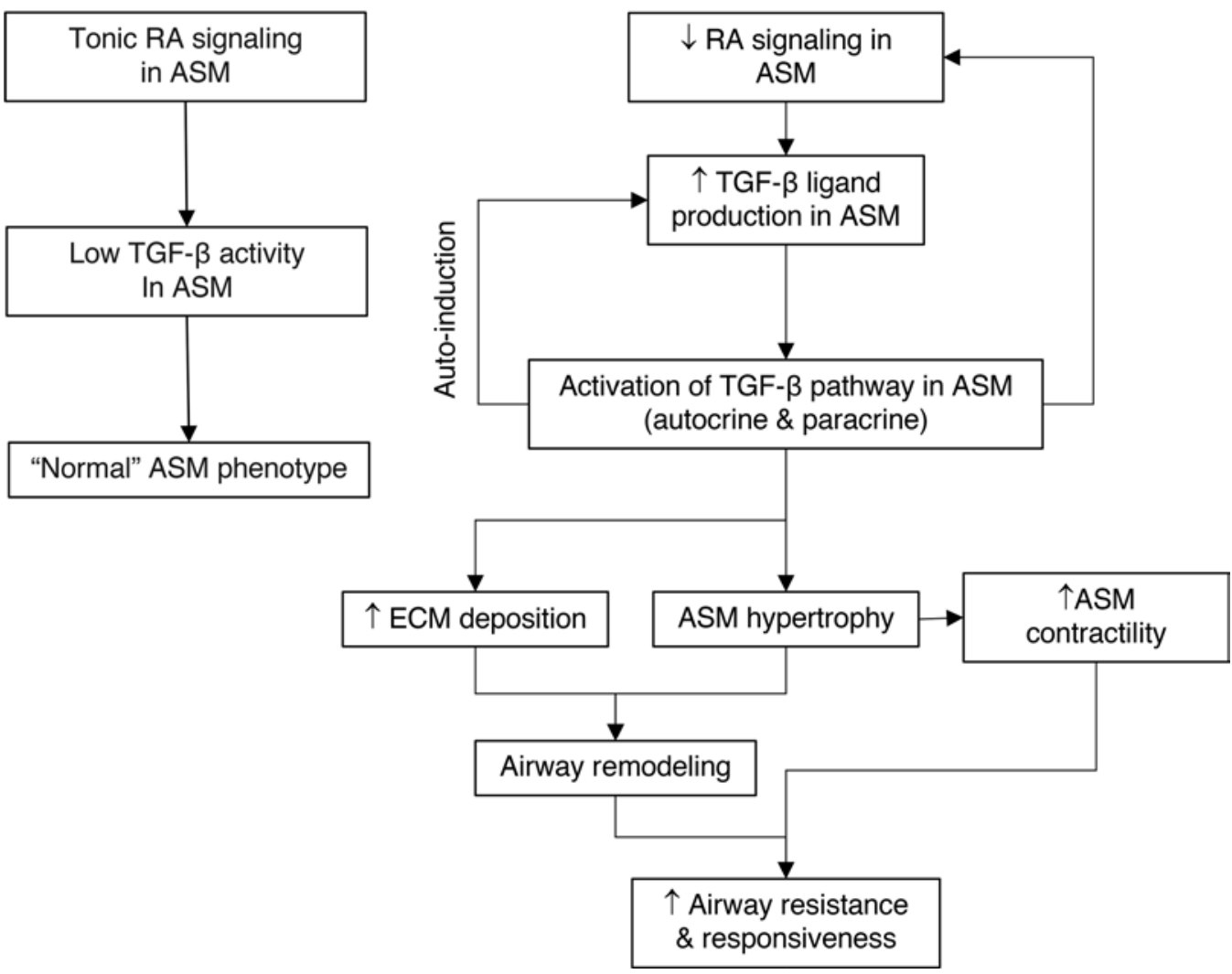

Figure 8. RA signaling in ASM maintains normal ASM phenotype by preventing excessive activation of the TGF- $\beta$ pathway. Left: Tonic RA signaling in ASM keeps TGF- $\beta$ activity in ASM at a low level to maintain normal ASM phenotype. Right: Disruption of RA signaling in ASM leads to increased TGF- $\beta$ ligand production and TCF- $\beta$ pathway activation, causing increased ECM deposition, ASM hypertrophy, ASM hypercontractility, and airway remodeling, ultimately resulting in higher airway resistance and AHR. Autoinduction of TCF- $\beta$ ligand and inhibition of RA receptor-mediated signaling by TCF- $\beta$ results in persistent TGF- $\beta$ activation.

Culture of hASM cells. These deidentified primary hASM cells, provided by the Gift of Hope Foundation, were isolated by enzymatic digestion from human donor lungs that were not suitable for transplantation (47). hASM cells from 2 unrelated adult donors without a known history of chronic lung diseases were used in our experiments. A frozen vial of hASM cells from each donor was thawed and cultured in $75-\mathrm{cm}^{2}$ culture flasks containing DMEM/F12 supplemented with $10 \%$ FBS and $1 \times$ antibiotic/antimycotic mixture to $80 \%$ confluence. The cells from each donor were then counted and split into 4 wells at 22,000 cells per well and cultured in CTR medium, or medium containing the pan-RA receptor inhibitor BMS $\left(10^{-6} \mathrm{M}\right.$; Sigma-Aldrich, B6688), the RA synthesis inhibitor DEAB (10-5 M; Sigma-Aldrich, D86256), or TGF- $\beta 1$ (20 ng/ $\mu 1$, R\&D Systems, 7754-BH-025/CF) for 24 hours, then processed for real-time PCR, microarray, or Western blot.

$P C R$. Total RNA was isolated using an RNeasy Mini Kit (Qiagen, 74106) and then reverse transcribed into cDNA (SuperScript III, Thermo Fisher Scientific, 18080051). For reverse transcription PCR (RT-PCR), cDNA was used as a template for 30 cycles of PCR using gene-specific primers (Supplemental Table 1). For real-time PCR, the gene of interest was amplified using cDNA and TaqMan probes (Thermo Fisher Scientific, 4331182). A dissociation curve was used to determine the relative concentration of the single PCR product. $\beta$-Actin mRNA (Actb; Thermo Fisher Scientific, 4352341E) was used as internal control. mASM cells from 6 mice per experimental condition were used for RT- and real-time PCR analyses. hASM cells from all 3 donors cultured in different conditions were used for real-time PCR analysis.

Western blot analysis. Protein extracts $(50 \mu \mathrm{g})$ from cultured hASM was subjected to SDS-PAGE, blotted onto nitrocellulose, incubated overnight with primary antibodies in Tris-buffered saline with $0.1 \%$ Tween-20 and 5\% milk (p-SMAD2, Cell Signaling Technology, 18338, 1:1,000; tSMAD2, Cell Signaling 
Technology, 5339, 1:1,000; $\alpha$-SMA, Sigma-Aldrich, A5228, 1:500; SM22 $\alpha$, Abcam, AB14106, 1:8,000; GAPDH, Cell Signaling Technology, 5174, 1:1,000), and developed (SuperSignal West Pico; Thermo Fisher Scientific, 34577). The blots were then quantified using ImageJ (NIH) (77). Results for $\alpha$-SMA and SM22 $\alpha$ were normalized to GAPDH. The p-SMAD2 result was normalized to tSMAD2 and GAPDH. The relative expression level was further normalized to CTR-treated hASM from the same donor.

Detection of lacZ/ $\beta$-gal/ activity (S-gal staining) in RARE-lacZ mice. Lungs from RARE-lacZ mice were fixed in $0.2 \%$ glutaraldehyde $/ 2 \%$ paraformaldehyde at $4^{\circ} \mathrm{C}$ for 20 minutes, followed by cryoprotection (30\% sucrose) and cryosections $(10 \mu \mathrm{m})$. The sections were washed with PBS, incubated with rinse buffer ( $0.1 \%$ sodium deoxycholate, $0.2 \%$ IGEPAL CA-630, $2 \mathrm{mM} \mathrm{MgCl}_{2}$, in PBS), and developed with S-gal substrate solution (1 mg/ml S-gal [Sigma-Aldrich, RES1364C-A101X], 0.4 mM NBT [4-nitro blue tetrazolium chloride; Roche, 11383213001], in rinse buffer) overnight at $37^{\circ} \mathrm{C}$ protected from light (46). Lungs from 5 $R A R E-l a c Z$ mice were used for the detection of RARE-lacZ signal.

Histology. For plastic sections, lungs were isolated, fixed in $2 \%$ glutaraldehyde $/ 1 \%$ paraformaldehyde in $0.1 \mathrm{M}$ sodium cacodylate buffer for 4 hours, embedded in JB4 resin, sectioned to $1 \mu \mathrm{m}$ thickness, then imaged. Trichrome staining was performed using a kit (Sigma-Aldrich, HT15) with protocols provided by the manufacturer. For immunohistochemistry, tissue sections were first incubated with primary antibodies ( $\alpha$-SMA, Sigma-Aldrich, A5228, 1:500; Ki-67, Abcam, AB15580, 1:500; GFP, Aves, GFP-1020, 1:500; p-SMAD2, Cell Signaling Technology, 18338, 1:500; $\beta$-gal, MP Biomedicals, 08A002720, 1:5,000; RARA, Abcam, 28767, 1:50; RARB, Abcam, AB53161, 1:100; RARG, Abcam, AB191368, 1:50), incubated with HRP- or fluorophore-conjugated secondary antibodies, then developed using kits and protocols provided by the manufacturer (Vector Laboratories). Three or more mice in each experimental condition were used for histologic analyses.

Morphometry. $\alpha$-SMA and GFP dual-immunostained lung from tamoxifen-injected Myh11-CreERT;mT/mG mice was used to quantify numbers of ASM in the airway. $\alpha$-SMA $\mathrm{GFP}^{+}$cells were counted in 25 large airways (lumen diameter $>400 \mu \mathrm{m}$ ) then normalized to the length of their basement membrane using ImageJ (77). To quantify the thickness of ASM layers, we used lung sections immunostained with $\alpha$-SMA. The margins of the ASM layer from 25 large airways (lumen diameter $>400 \mu \mathrm{m}$ ) were outlined using ImageJ, and the thickness was determined by drawing a perpendicular line from the basement membrane to the outer margin of the ASM layer. The ASM layer thicknesses of RA-sufficient and RA-deficient airways of equal diameter were then compared. Trichrome-stained lung sections were used to quantify ECM volumefraction in the ASM layer. The margins of ASM layer were outlined as described above and the regions stained blue (collagen fibers) within the ASM layer were determined using ImageJ software. The ECMfraction \% was determined by taking the area stained blue divided by the total area of the ASM layer in each airway. Twenty-five large airways (lumen diameter $>400 \mu \mathrm{m}$ ) and 25 medium/small airways (lumen diameter $150-400 \mu \mathrm{m}$ ) from each experimental group were used to quantify ECM-fraction \%. More than 3 mice from each experimental group were used for morphometric analyses. To determine hASM cell size, a photomicrograph of the cultured cells was taken at $\times 100$ magnification after 24 hours of culture. ImageJ software was used to outline and quantify the area of each cell. One hundred cells per culture condition per donor were used to assess cell size.

Microarray analysis. For the analysis of mASM, we isolated total RNA from freshly sorted mASM from VAS, VAD, CTR, and BMS lungs using an RNeasy Mini Kit; dietary and pharmacologic modulations of RA activity were only performed for 3 days here to detect early RA targets. Because we could obtain only approximately 30 ng of RNA per mouse, we had to pool mASM from 3 mice per experimental group to generate an informative mRNA profile. Mouse Gene 2.0 ST arrays (Affymetrix, 4 chips total) were used to produce mRNA profiles from each group. Gene expression profiles were compared between VAS and VAD mASM, and CTR and BMS mASM. Genes induced ( $\geq 1.3$-fold increase) in both models of RA deficiency were considered significant. For the analysis of hASM, we isolated total RNA from hASM from all 3 donors cultured in CTR and BMS-containing media for 24 hours using the RNeasy Mini Kit. Human Gene 2.0 ST arrays (9 chips total) were used to generate mRNA profiles from CTR and BMS-treated hASM from each donor. Expression profiles from CTR and BMS-treated hASM from each donor were compared. Genes that were upregulated by BMS treatment (false discovery rate $<0.25$ ) were deemed RA targets. To generate the final list of potential RA targets, we included only genes induced in mASM from both models and in human ASM. This list was then loaded into EASE software, which used Fisher's exact test to detect enriched gene sets $(58,59)$. All microarray data have been submitted to NCBI's Gene Expression Omnibus (GEO GSE116845 and GSE116846). 
Measurement of hASM contraction. The contractile force screening approach (52) entailed 3 sequential steps: (a) Preparation of custom 96-well plates. Plate fabrication consisted of applying a $100-\mu \mathrm{m}$-thick layer of deformable substrate (NuSil 8100, NuSil, Young's modulus $=9.45 \mathrm{kPa}$ ), adding fiduciary fluorescent beads (diameter $=\sim 700 \mathrm{~nm}$ ) to reveal cell-induced substrate deformation, adding 96-well dividers to create a multiwell environment, and finally binding $30 \mu \mathrm{g} / \mathrm{ml}$ monomeric collagen (PureCol) diluted in PBS to the substrate to facilitate cell adhesion. (b) Measurement of hASM contractile force. Cells were plated as confluent monolayers at a density of 22,000 cells per well. One day after plating, they were treated with drug or control for an additional 24 hours and prepared for measurements. Fluorescent beads directly underneath the cells were microscopically observed (DMI 6000B, Leica Inc., $\times 10$ objective) and recorded (Leica DFC365 FX camera), with the cells adherent, and after the cells were detached. Using the relative bead movement (Matlab, Mathworks Inc.), the predefined substrate stiffness, and thickness, traction force maps were computed on a well-by-well basis using the approach of Fourier-transform traction cytometry (78) modified to the case of cell monolayers $(79,80)$. From the traction force map and the corresponding bead displacements, we computed a single scalar metric called the strain energy that represents the total work done by the cells on their substrate because of contraction. This parameter is a well-established quantitative measure of overall cell contraction (78). (c) Presentation of results. Each treatment was performed in 9 or 10 separate wells. The strain energy was averaged across these wells and further normalized to the average value for control cells tested under identical experimental conditions. The measurements were repeated in cells isolated from 3 human donor lungs.

$\mathrm{MeCh}$ challenge and airway resistance measurement. Adult mice were sedated, anesthetized, and paralyzed (i.p. xylazine $10 \mathrm{mg} / \mathrm{kg}$ BW; i.p. pentobarbital $100 \mathrm{mg} / \mathrm{kg}$ BW; i.p. pancuronium $0.5 \mathrm{mg} / \mathrm{kg} \mathrm{BW}$ ), intubated, placed on a mechanical ventilator (Legacy flexiVent, SCIREQ), and ventilated at $300 \mathrm{breaths} / \mathrm{min}$ (tidal volume $6-7 \mathrm{ml} / \mathrm{kg} \mathrm{BW} ; 3 \mathrm{cmH}_{2} \mathrm{O}$ positive end-expiratory pressure). Peak airway resistance was measured after airway delivery of nebulized vehicle $(0 \mathrm{mg} / \mathrm{ml} \mathrm{MeCh}$ in PBS) and delivery of increasing concentrations of nebulized $\mathrm{MeCh}(5 \mathrm{mg} / \mathrm{ml}, 10 \mathrm{mg} / \mathrm{ml}$, and $15 \mathrm{mg} / \mathrm{ml}$, in PBS). flexiVent data are presented as absolute or relative peak resistance to the baseline airway resistance of the control (VAS, CTR, OILCTR, or CTR-28R depending on the experiment). Nine mice were used for each experimental condition where CD1 or RARElacZ mice were used; 6 male mice per experimental condition were used to measure airway resistance involving $M y h 11-C r e E R T 2 ; T g f b r 2^{f / f l}$ mice.

Tamoxifen injection. Cre-Lox recombination in Myh11-CreERT2;mT/mG and Myh11-CreERT2;Tgfbr $2^{f l / f l}$ male mice was induced by tamoxifen (Sigma-Aldrich, T5648) at $1 \mathrm{mg} /$ day i.p. for 5 days. These mice were then given control (TAM-CTR) or BMS-containing (TAM-BMS) diet 28 days after the last i.p. injection. Vehicle-injected (corn oil) litter mates followed by 5 days of CTR and BMS-containing diet were used as additional controls (OIL-CTR and OIL-BMS).

Statistics. Scatter plots (Figure 1A; Figure 2, D and E; Figure 3, B, D, and F; Figure 4, C-E; Figure 5, A-D; Figure 6E; Figure 7B; and Supplemental Figures 1, 2, and 5) were graphed as mean \pm SEM. Dose-response curves (Figure 1, B and C; Figure 6F; Figure 7C) were plotted as mean \pm range. In each box-and-whisker plot (Figure 3, A and C), the box was bounded on the top by the third quartile and the bottom by the first quartile and were divided by the median; the top whiskers extended up from the third quartile to the maximum value and the lower whiskers extended downward from the first quartile to the minimum value. Student's $t$ test was used for statistical analysis for data presented in Figure 1A; Figure 2D; Figure 3, A and B; Figure 4D; and Figure 7, B and C. Bonferroni's correction was applied to data analysis involving multiple comparisons (Figure 2E; Figure 3, C, D, and F; Figure 4, C and E; Figure 5, A-D; Figure 6C; and Supplemental Figure 5, B and C). Two-way ANOVA was used to compare dose-response curves (Figure 1, B and C; Figure 6F; Figure 7C; and Supplemental Figure 1, A-C; Supplemental Figure 2, A-C; and Supplemental Figure 5A). Differences were considered significant if the $P$ value (or the Bonferroni-adjusted $P$ value) was less than 0.05 .

Study approval. All experiments involving animals were performed according to protocols approved by the IACUC of the Boston University Medical Campus, Boston, MA.

\section{Author contributions}

All authors participated in the design, execution, and interpretation of the experiments. FC, RK, and AF conceived and designed the study. FC wrote the manuscript. FC and FZS performed most experiments and data analysis. RK, SY, and SRM performed experiments and data analysis involving contraction force screening. TN contributed to the design and interpretation of the study. 


\section{Acknowledgments}

We thank the Boston University Microarray and Sequencing Resource Core, Boston University Flow Cytometry Core, and SCIREQ for technical assistance. We are grateful to the Gift of Hope Foundation for providing primary ASM from human lung donors, and to David M. Center, Joseph P. Mizgerd, and Frederic F. Little for critical reading of this manuscript. This work was supported by grants from NIH/NHLBI (R01 HL 116163, R01 HL 130287) and NIH/NCATS (UL1-TR001430).

Address correspondence to: Felicia Chen, The Pulmonary Center, Boston University School of Medicine, 72 East Concord Street, R-304, Boston, Massachusetts 02118, USA. Phone: 617.358.1197; Email: felichen@bu.edu.

1. Amrani Y, Panettieri RA. Airway smooth muscle: contraction and beyond. Int J Biochem Cell Biol. 2003;35(3):272-276

2. Lauzon AM, Martin JG. Airway hyperresponsiveness; smooth muscle as the principal actor. F1000Res. 2016;5.

3. Bumbacea D, et al. Parameters associated with persistent airflow obstruction in chronic severe asthma. Eur Respir J. 2004;24(1):122-128

4. McKeever TM, Lewis SA, Smit HA, Burney P, Cassano PA, Britton J. A multivariate analysis of serum nutrient levels and lung function. Respir Res. 2008;9:67.

5. Amaral CT, et al. Vitamin A deficiency alters airway resistance in children with acute upper respiratory infection. Pediatr Pulmonol. 2013;48(5):481-489.

6. Allen S, Britton JR, Leonardi-Bee JA. Association between antioxidant vitamins and asthma outcome measures: systematic review and meta-analysis. Thorax. 2009;64(7):610-619.

7. Lin YC, Wu TC, Chen PY, Hsieh LY, Yeh SL. Comparison of plasma and intake levels of antioxidant nutrients in patients with chronic obstructive pulmonary disease and healthy people in Taiwan: a case-control study. Asia Pac J Clin Nutr. 2010;19(3):393-401.

8. Rivas-Crespo MF, et al. High serum retinol and lung function in young patients with cystic fibrosis. J Pediatr Gastroenterol Nutr. 2013;56(6):657-662.

9. Soler Artigas M, et al. Genome-wide association and large-scale follow up identifies 16 new loci influencing lung function. Nat Genet. 2011;43(11):1082-1090.

10. Collins SA, et al. HHIP, HDAC4, NCR3 and RARB polymorphisms affect fetal, childhood and adult lung function. Eur Respir J. 2013;41(3):756-757.

11. Li X, et al. Genome-wide association study identifies TH1 pathway genes associated with lung function in asthmatic patients. J Allergy Clin Immunol. 2013;132(2):313-20.e15.

12. Wilk JB, et al. Genome-wide association studies identify CHRNA5/3 and HTR4 in the development of airflow obstruction. Am J Respir Crit Care Med. 2012;186(7):622-632.

13. Theodosiou M, Laudet V, Schubert M. From carrot to clinic: an overview of the retinoic acid signaling pathway. Cell Mol Life Sci. 2010;67(9):1423-1445.

14. Godinas L, Corhay JL, Henket M, Guiot J, Louis R, Moermans C. Increased production of TGF- $\beta 1$ from sputum cells of COPD: Relationship with airway obstruction. Cytokine. 2017;99:1-8.

15. Redington AE, et al. Transforming growth factor-beta 1 in asthma. Measurement in bronchoalveolar lavage fluid. Am J Respir Crit Care Med. 1997;156(2 Pt 1):642-647.

16. Vignola AM, et al. Transforming growth factor-beta expression in mucosal biopsies in asthma and chronic bronchitis. Am $J$ Respir Crit Care Med. 1997;156(2 Pt 1):591-599.

17. Oenema TA, et al. Muscarinic receptor stimulation augments TGF- $\beta 1$-induced contractile protein expression by airway smooth muscle cells. Am J Physiol Lung Cell Mol Physiol. 2012;303(7):L589-L597.

18. Bossé Y, Stankova J, Rola-Pleszczynski M. Transforming growth factor-beta1 in asthmatic airway smooth muscle enlargement: is fibroblast growth factor-2 required? Clin Exp Allergy. 2010;40(5):710-724.

19. Goldsmith AM, et al. Transforming growth factor-beta induces airway smooth muscle hypertrophy. Am J Respir Cell Mol Biol. 2006;34(2):247-254

20. Gawaziuk JP, Sheikh F, Cheng ZQ, Cattini PA, Stephens NL. Transforming growth factor-beta as a differentiating factor for cultured smooth muscle cells. Eur Respir J. 2007;30(4):643-652.

21. Oliver MN, Fabry B, Marinkovic A, Mijailovich SM, Butler JP, Fredberg JJ. Airway hyperresponsiveness, remodeling, and smooth muscle mass: right answer, wrong reason? Am J Respir Cell Mol Biol. 2007;37(3):264-272.

22. Benayoun L, Druilhe A, Dombret MC, Aubier M, Pretolani M. Airway structural alterations selectively associated with severe asthma. Am J Respir Crit Care Med. 2003;167(10):1360-1368.

23. Ojiaku CA, et al. TGF- $\beta 1$ evokes human airway smooth muscle cell shortening and hyperresponsiveness via Smad3. Am J Respir Cell Mol Biol. 2018;58(5):575-584.

24. Verrecchia F, Chu ML, Mauviel A. Identification of novel TGF-beta /Smad gene targets in dermal fibroblasts using a combined cDNA microarray/promoter transactivation approach. J Biol Chem. 2001;276(20):17058-17062.

25. Chen F, et al. Prenatal retinoid deficiency leads to airway hyperresponsiveness in adult mice. J Clin Invest. 2014;124(2):801-811.

26. Kim YK, et al. Retinyl ester formation by lecithin: retinol acyltransferase is a key regulator of retinoid homeostasis in mouse embryogenesis. J Biol Chem. 2008;283(9):5611-5621.

27. van de Pavert SA, et al. Maternal retinoids control type 3 innate lymphoid cells and set the offspring immunity. Nature. 2014;508(7494):123-127.

28. Chen F, Cao Y, Qian J, Shao F, Niederreither K, Cardoso WV. A retinoic acid-dependent network in the foregut controls formation of the mouse lung primordium. J Clin Invest. 2010;120(6):2040-2048. 
29. Rossant J, Zirngibl R, Cado D, Shago M, Giguère V. Expression of a retinoic acid response element-hsplacZ transgene defines specific domains of transcriptional activity during mouse embryogenesis. Genes Dev. 1991;5(8):1333-1344.

30. Vanoirbeek JA, et al. Noninvasive and invasive pulmonary function in mouse models of obstructive and restrictive respiratory diseases. Am J Respir Cell Mol Biol. 2010;42(1):96-104.

31. Halwani R, Al-Muhsen S, Hamid Q. Airway remodeling in asthma. Curr Opin Pharmacol. 2010;10(3):236-245.

32. Brown RH, Pearse DB, Pyrgos G, Liu MC, Togias A, Permutt S. The structural basis of airways hyperresponsiveness in asthma. J Appl Physiol. 2006;101(1):30-39.

33. Baroffio M, Barisione G, Crimi E, Brusasco V. Noninflammatory mechanisms of airway hyper-responsiveness in bronchial asthma: an overview. Ther Adv Respir Dis. 2009;3(4):163-174.

34. Porsbjerg C, Brannan JD, Anderson SD, Backer V. Relationship between airway responsiveness to mannitol and to methacholine and markers of airway inflammation, peak flow variability and quality of life in asthma patients. Clin Exp Allergy. 2008;38(1):43-50

35. Jeffery PK, Wardlaw AJ, Nelson FC, Collins JV, Kay AB. Bronchial biopsies in asthma. An ultrastructural, quantitative study and correlation with hyperreactivity. Am Rev Respir Dis. 1989;140(6):1745-1753.

36. Hargreave FE, Dolovich J, O'Byrne PM, Ramsdale EH, Daniel EE. The origin of airway hyperresponsiveness. J Allergy Clin Immunol. 1986;78(5 Pt 1):825-832.

37. Dolovich J, Hargreave F. The asthma syndrome: inciters, inducers, and host characteristics. Thorax. 1981;36(9):614-644.

38. Israel E, Reddel HK. Severe and Difficult-to-Treat Asthma in Adults. N Engl J Med. 2017;377(10):965-976.

39. McGowan SE. Vitamin A deficiency increases airway resistance following C-fiber stimulation. Respir Physiol Neurobiol. 2007;157(2-3):281-289.

40. McGowan SE, Takle EJ, Holmes AJ. Vitamin A deficiency alters the pulmonary parenchymal elastic modulus and elastic fiber concentration in rats. Respir Res. 2005;6:77.

41. McGowan SE, Holmes AJ, Smith J. Retinoic acid reverses the airway hyperresponsiveness but not the parenchymal defect that is associated with vitamin A deficiency. Am J Physiol Lung Cell Mol Physiol. 2004;286(2):L437-L444.

42. McGowan SE, et al. Vitamin A deficiency promotes bronchial hyperreactivity in rats by altering muscarinic $\mathrm{M}(2)$ receptor function. Am J Physiol Lung Cell Mol Physiol. 2002;282(5):L1031-L1039.

43. Paez-Cortez J, et al. A new approach for the study of lung smooth muscle phenotypes and its application in a murine model of allergic airway inflammation. PLoS One. 2013;8(9):e74469.

44. Minoo P, Su G, Drum H, Bringas P, Kimura S. Defects in tracheoesophageal and lung morphogenesis in Nkx2.1(-/-) mouse embryos. Dev Biol. 1999;209(1):60-71.

45. Ghosh S, et al. Activation dynamics and signaling properties of Notch3 receptor in the developing pulmonary artery. $J$ Biol Chem. 2011;286(25):22678-22687.

46. Merkwitz C, Blaschuk O, Winkler J, Schulz A, Prömel S, Ricken AM. Advantages and limitations of salmon-gal/tetrazolium salt histochemistry for the detection of LacZ reporter gene activity in murine epithelial tissue. J Histochem Cytochem. 2017;65(4):197-206

47. Panettieri RA. Isolation and culture of human airway smooth muscle cells. Methods Mol Med. 2001;56:155-160.

48. Day RM, Lee YH, Park AM, Suzuki YJ. Retinoic acid inhibits airway smooth muscle cell migration. Am J Respir Cell Mol Biol. 2006;34(6):695-703.

49. Russo JE, Hauguitz D, Hilton J. Inhibition of mouse cytosolic aldehyde dehydrogenase by 4-(diethylamino)benzaldehyde. Biochem Pharmacol. 1988;37(8):1639-1642.

50. Wright DB, et al. Phenotype modulation of airway smooth muscle in asthma. Pulm Pharmacol Ther. 2013;26(1):42-49.

51. Manabe I, Nagai R. Regulation of smooth muscle phenotype. Curr Atheroscler Rep. 2003;5(3):214-222.

52. Park CY, et al. High-throughput screening for modulators of cellular contractile force. Integr Biol (Camb). 2015;7(10):1318-1324

53. Gerdes J, Schwab U, Lemke H, Stein H. Production of a mouse monoclonal antibody reactive with a human nuclear antigen associated with cell proliferation. Int J Cancer. 1983;31(1):13-20.

54. Miano JM, Cserjesi P, Ligon KL, Periasamy M, Olson EN. Smooth muscle myosin heavy chain exclusively marks the smooth muscle lineage during mouse embryogenesis. Circ Res. 1994;75(5):803-812.

55. Wirth A, et al. G12-G13-LARG-mediated signaling in vascular smooth muscle is required for salt-induced hypertension. Nat Med. 2008;14(1):64-68.

56. Groneberg D, König P, Wirth A, Offermanns S, Koesling D, Friebe A. Smooth muscle-specific deletion of nitric oxide-sensitive guanylyl cyclase is sufficient to induce hypertension in mice. Circulation. 2010;121(3):401-409.

57. Muzumdar MD, Tasic B, Miyamichi K, Li L, Luo L. A global double-fluorescent Cre reporter mouse. Genesis. 2007;45(9):593-605

58. Huang da W, Sherman BT, Lempicki RA. Systematic and integrative analysis of large gene lists using DAVID bioinformatics resources. Nat Protoc. 2009;4(1):44-57.

59. Huang da W, Sherman BT, Lempicki RA. Bioinformatics enrichment tools: paths toward the comprehensive functional analysis of large gene lists. Nucleic Acids Res. 2009;37(1):1-13.

60. Massagué J. TGFß signalling in context. Nat Rev Mol Cell Biol. 2012;13(10):616-630.

61. Xi H, Shuai QG, Shao LL. Involvement of the TGF $\beta 1 /$ Smad2/MMP3 signaling pathway in SB431542-induced inhibition of cell invasion in multiple myeloma RPMI 8226 cells. Oncol Lett. 2017;14(1):541-546.

62. Levéen $\mathrm{P}$, et al. Induced disruption of the transforming growth factor beta type II receptor gene in mice causes a lethal inflammatory disorder that is transplantable. Blood. 2002;100(2):560-568.

63. Li W, et al. Tgfbr2 disruption in postnatal smooth muscle impairs aortic wall homeostasis. J Clin Invest. 2014;124(2):755-767.

64. Williams SR. Nutrition and Diet Therapy. St. Louis, MO: Mosby; 1997.

65. Conrad Z, Johnson LK, Juan W, Roemmich JN, Jahns L. Nutrient inadequacy among nutritionally vulnerable populations in the US. FASEB Journal. 2017;31(1_Suppl):445.1.

66. Trasino SE, Tang XH, Jessurun J, Gudas LJ. Obesity leads to tissue, but not serum vitamin A deficiency. Sci Rep. $2015 ; 5: 15893$.

67. Clugston RD, Blaner WS. The adverse effects of alcohol on vitamin A metabolism. Nutrients. 2012;4(5):356-371.

68. Matheu V, et al. Impact on allergic immune response after treatment with vitamin A. Nutr Metab (Lond). $2009 ; 6: 44$. 
69. Wu J, Zhang Y, Liu Q, Zhong W, Xia Z. All-trans retinoic acid attenuates airway inflammation by inhibiting Th2 and Th17 response in experimental allergic asthma. BMC Immunol. 2013;14:28.

70. Hu B, Wu Z, Phan SH. Smad3 mediates transforming growth factor-beta-induced alpha-smooth muscle actin expression. Am J Respir Cell Mol Biol. 2003;29(3 Pt 1):397-404.

71. Li K, et al. The study of the ratio and distribution of Th17 cells and Tc17 cells in asthmatic patients and the mouse model. Asian Pac J Allergy Immunol. 2013;31(2):125-131.

72. Barczyk A, Pierzchala W, Sozañska E. Interleukin-17 in sputum correlates with airway hyperresponsiveness to methacholine. Respir Med. 2003;97(6):726-733.

73. Reynolds JM, et al. Interleukin-17B antagonizes interleukin-25-mediated mucosal inflammation. Immunity. 2015;42(4):692-703.

74. Kim SJ, et al. Autoinduction of transforming growth factor beta 1 is mediated by the AP-1 complex. Mol Cell Biol. 1990;10(4):1492-1497.

75. Van Obberghen-Schilling E, Roche NS, Flanders KC, Sporn MB, Roberts AB. Transforming growth factor beta 1 positively regulates its own expression in normal and transformed cells. J Biol Chem. 1988;263(16):7741-7746.

76. Zhu X, Bergles DE, Nishiyama A. NG2 cells generate both oligodendrocytes and gray matter astrocytes. Development. 2008;135(1):145-157.

77. Schneider CA, Rasband WS, Eliceiri KW. NIH Image to ImageJ: 25 years of image analysis. Nat Methods. 2012;9(7):671-675.

78. Butler JP, Toli冈-Nørrelykke IM, Fabry B, Fredberg JJ. Traction fields, moments, and strain energy that cells exert on their surroundings. Am J Physiol, Cell Physiol. 2002;282(3):C595-C605.

79. Kim JH, et al. Propulsion and navigation within the advancing monolayer sheet. Nat Mater. 2013;12(9):856-863.

80. Tambe DT, et al. Collective cell guidance by cooperative intercellular forces. Nat Mater. 2011;10(6):469-475. 